\title{
A 4-node 3D-shell element to model shell surface tractions and incompressible behavior
}

\author{
Do-Nyun Kim, Klaus-Jürgen Bathe* \\ Department of Mechanical Engineering, Massachusetts Institute of Technology, Cambridge MA 02139 United States
}

\section{A R T I C L E I N F O}

\section{Article history:}

Received 19 February 2008

Accepted 16 April 2008

Available online 7 July 2008

\section{Keywords:}

Shells

MITC4 element

3D-shell model

Surface tractions

Incompressible analysis

Ill-conditioning

\begin{abstract}
A B S T R A C T
We present in this paper a shell element that models the three-dimensional (3D) effects of surface tractions, like needed when a shell is confined between other solid media. The element is the widely used MITC4 shell element enriched by the use of a fully 3D stress-strain description, appropriate throughthe-thickness displacements to model surface tractions, and pressure degrees of freedom for incompressible analyses. The element formulation avoids instabilities and ill-conditioning. Various example solutions are presented to illustrate the capabilities of the element.
\end{abstract}

(c) 2008 Elsevier Ltd. All rights reserved.

\section{Introduction}

The finite element analysis of shells has now matured to the extent that it sometimes seems any complex shell can be accurately analyzed. In fact, however, there are still a number of outstanding difficulties. These relate to the more accurate modeling of shell structures to include 3D effects, the development of still more effective shell finite elements, in particular triangular elements, and the mathematical analyses to ensure optimality of the finite element solutions $[1,2]$. To a large extent, shell structures are now solved in practice using 4-node shell elements. Among those available, the MITC4 shell element is probably the most effective element, in particular when linear and nonlinear analyses are to be performed [1-6]. However, the other MITC shell elements can be more effective for specific analyses [5,7-9].

The formulation of the MITC4 shell element is based on the Reissner-Mindlin assumptions of "material fibers originally straight and normal to the shell mid-surface do not stretch and remain straight" and "zero stress normal to the shell mid-surface" $[1,2]$. The MITC elements can be formulated using a continuum representation or the 'basic shell model' identified by Chapelle and Bathe [1,2,10,11]. While the Reissner-Mindlin assumptions are widely applicable, they cannot model the application of shell surface stresses as needed in the analysis of a shell confined between solid media. For example, in metal forming, the normal

\footnotetext{
* Corresponding author.

E-mail address: KJB@mit.edu (K.J. Bathe).
}

and shear tractions applied to thin sheet surfaces can be large and the accurate modeling of normal stresses and shear stresses can be important. Of course, in addition, large strain elastic-plastic (almost incompressible) conditions need be represented.

To model the effects of surface tractions, it appears that 3D shell-solid elements are most appropriate, see [12-16] and the references therein. In the formulation of these elements, the top and bottom surfaces of the shell are represented geometrically and their positions are updated through the displacement degrees of freedom, just like in a fully 3D analysis of solids but with only one element layer through the shell thickness [1,2]. Since the bending strains vary linearly, clearly, for consistency with bending theory including the Poisson ratio coupling, the through-the-thickness normal strain must also be allowed to vary linearly. This requires a quadratic interpolation of displacements through the shell thickness. The shear, membrane and pinching locking can be relieved using MITC strain interpolations.

While sometimes effective, these elements display severe illconditioning when the shell becomes thin and when the shell is an (almost) incompressible medium $[17,18]$. To improve the element behavior and computational effectiveness, instead of displacement degrees of freedom, enhanced strains have been used, see for example Refs. $[12,19,20]$, and the references therein. However, enhanced strain formulations can be unstable, see Refs. $[21,22]$, and are therefore best avoided. Hence, a more effective approach is to build on the basic MITC4 shell element, for which all shell actions are represented using the shell mid-surface, use the three-dimensional stress-strain description, and enrich the shell 
element formulation 'judiciously' by appropriate displacement and pressure interpolations. These interpolations need to be selected to not introduce instabilities or ill-conditioning. This approach was used in Ref. [23] to enrich the MITC4 shell element by two element degrees of freedom corresponding to thickness stretching (a constant and a linear term) in order to represent large strain effects.

The objective in this paper is to present the MITC4 shell element enriched by the 3D stress-strain law, and by displacements and pressure used to represent accurately the effects of shell surface normal and tangential tractions, and incompressible conditions. The enrichment in displacements is achieved by simply adding displacement interpolations with corresponding degrees of freedom at the four nodes. The resulting displacements are compatible across element boundaries. To render the element also applicable to (almost) incompressible conditions, the $u / p$ formulation with an assumption on the pressure is used [1]. All these degrees of freedom, including the pressure degrees of freedom, can be invoked hierarchically as desired. This hierarchical feature of the element is very attractive, obviously from a modeling point of view, but also from a practical point of view. Namely, the pre- and post-processing capabilities of the classical MITC4 element are directly applicable by simply increasing the number of degrees of freedom allowed for the element.

The element presented in this paper is largely based on concepts previously published but in this paper we have synthesized various ideas in an aim to obtain an overall effective formulation. In particular, this 3D-shell element formulation does not show ill-conditioning. In the following sections, we first present the formulation of the continuum shell model, then the finite element discretization, and finally we give the results of various illustrative example solutions.

\section{The shell model}

In this section, we present the shell model that we will solve by our finite element discretization. We follow the notation used in Ref. [1].

The initial geometry at time 0 is described by

${ }^{0} \underline{\boldsymbol{x}}={ }^{0} \underline{\boldsymbol{x}}_{M}+\frac{\xi}{2}{ }^{0} a^{0} \underline{\boldsymbol{V}}_{n}$,

where $\xi$ is the natural coordinate in the thickness direction, ${ }^{0} \underline{\boldsymbol{x}}_{M}$ is the position vector of material particles in the shell mid-surface, ${ }^{0} a$ is the thickness and ${ }^{0} \underline{V}_{n}$ is the director vector.

In the deformed configuration, an initially straight fiber may be curved with the assumption shown in Figs. 1 and 2. The assumed deformed geometry at time $t$ is

$$
\begin{aligned}
{ }^{t} \underline{\boldsymbol{x}}= & { }^{t} \underline{\boldsymbol{x}}_{M}+\frac{\xi}{2}{ }^{t} a^{t} \underline{\boldsymbol{V}}_{n}+\xi^{2}{ }^{0} a^{t} Q_{n}{ }^{t} \underline{\boldsymbol{V}}_{n}+\xi^{2}{ }^{0} a\left({ }^{t} Q_{1}{ }^{t} \underline{\boldsymbol{V}}_{1}+{ }^{t} Q_{2}{ }^{t} \underline{\boldsymbol{V}}_{2}\right) \\
& +\xi^{3}{ }^{0} a\left({ }^{t} C_{1}{ }^{t} \underline{\boldsymbol{V}}_{1}+{ }^{t} C_{2}{ }^{t} \underline{\boldsymbol{V}}_{2}\right) .
\end{aligned}
$$

Here ${ }^{t} \underline{\boldsymbol{V}}_{n}$ is the director vector at time $t,{ }^{t} a$ is the shell thickness at time $t,{ }^{t} \underline{V}_{\alpha}$, with $\alpha=1,2$ are unit vectors normal to ${ }^{t} \underline{\boldsymbol{V}}_{n},{ }^{t} Q_{n}$ is the degree of freedom corresponding to a quadratic displacement function in the direction ${ }^{t} \underline{\boldsymbol{V}}_{n}$, and ${ }^{t} Q_{\alpha}$ and ${ }^{t} C_{\alpha}$ represent degrees of freedom corresponding to quadratic and cubic displacement functions in the directions ${ }^{t} \underline{V} \alpha$, with $\alpha=1,2$. The left superscripts 0 and $t$ always denote that a quantity is given at times 0 and $t$. With these assumptions, the fiber
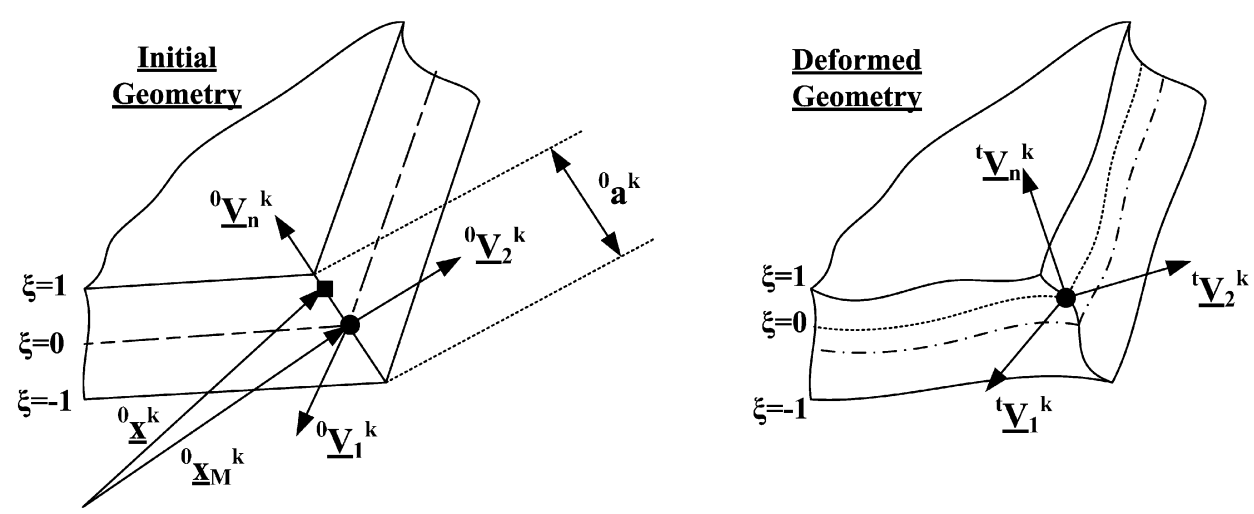

Fig. 1. Shell geometries in the initial and deformed configurations.
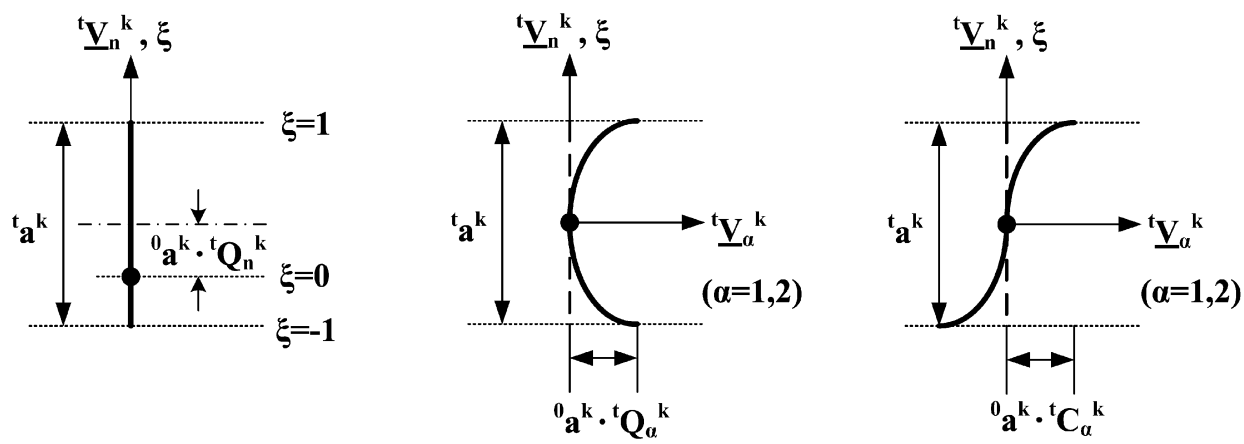

Fig. 2. The quadratic and cubic displacement functions at node $k$. 
originally normal to the mid-surface can strain linearly and become curved, and the transverse shear stresses have a quadratic distribution through the shell thickness. This enables the element to precisely capture the effect of surface tractions, like normal and frictional forces, applied on the top and bottom shell surfaces.

Note that ${ }^{0} a$, the initial thickness, is employed in front of each higher order function. This is a scaling factor in order to avoid illconditioning of the global stiffness matrix as the thickness becomes small. It is more natural to use the current thickness but we use the initial thickness because this leads to a simpler formulation.

In the same way, the deformed geometry at time $t+\Delta t$ is defined by

$$
\begin{aligned}
{ }^{t+\Delta t} \underline{\boldsymbol{x}}= & { }^{t+\Delta t} \underline{\boldsymbol{x}}_{M}+\frac{\xi}{2}{ }^{t+\Delta t} a^{t+\Delta t} \underline{\boldsymbol{V}}_{n}+\xi^{2}{ }^{0} a^{t+\Delta t} Q_{n}{ }^{t+\Delta t} \underline{\boldsymbol{V}}_{n} \\
& +\xi^{2}{ }^{0} a\left({ }^{t+\Delta t} Q_{1}{ }^{t+\Delta t} \underline{\boldsymbol{V}}_{1}+{ }^{t+\Delta t} Q_{2}{ }^{t+\Delta t} \underline{\boldsymbol{V}}_{2}\right) \\
& +\xi^{3}{ }^{0} a\left({ }^{t+\Delta t} C_{1}{ }^{t+\Delta t} \underline{\boldsymbol{V}}_{1}+{ }^{t+\Delta t} C_{2}{ }^{t+\Delta t} \underline{\boldsymbol{V}}_{2}\right) .
\end{aligned}
$$

From these geometric positions, we can obtain the corresponding incremental displacement field as follows:

$$
\begin{aligned}
\underline{\boldsymbol{u}} & ={ }^{t+\Delta t} \underline{\boldsymbol{u}}-{ }^{t} \underline{\boldsymbol{u}}={ }^{t+\Delta t} \underline{\boldsymbol{x}}-{ }^{t} \underline{\boldsymbol{x}}, \\
\underline{\boldsymbol{u}} & =\left({ }^{t+\Delta t} \underline{\boldsymbol{x}}_{M}-{ }^{t} \underline{\boldsymbol{x}}_{M}\right)+\frac{\xi}{2}\left({ }^{t+\Delta t} a^{t+\Delta t} \underline{\boldsymbol{V}}_{n}-{ }^{t} a^{t} \underline{\boldsymbol{V}}_{n}\right) \\
& +\xi^{2}{ }^{0} a\left({ }^{t+\Delta t} Q_{n}{ }^{t+\Delta t} \underline{\boldsymbol{V}}_{n}-{ }^{t} Q_{n}{ }^{t} \underline{\boldsymbol{V}}_{n}\right) \\
& +\xi^{2}{ }^{0} a\left({ }^{t+\Delta t} Q_{1}{ }^{t+\Delta t} \underline{\boldsymbol{V}}_{1}-{ }^{t} Q_{1}{ }^{t} \underline{\boldsymbol{V}}_{1}\right) \\
& +\xi^{2}{ }^{0} a\left({ }^{t+\Delta t} Q_{2}{ }^{t+\Delta t} \underline{\boldsymbol{V}}_{2}-{ }^{t} Q_{2}{ }^{t} \underline{\boldsymbol{V}}_{2}\right) \\
& +\xi^{3}{ }^{0} a\left({ }^{t+\Delta t} C_{1}{ }^{t+\Delta t} \underline{\boldsymbol{V}}_{1}-{ }^{t} C_{1}{ }^{t} \underline{\boldsymbol{V}}_{1}\right) \\
& +\xi^{3}{ }^{0} a\left({ }^{t+\Delta t} C_{2}{ }^{t+\Delta t} \underline{\boldsymbol{V}}_{2}-{ }^{t} C_{2}{ }^{t} \underline{\boldsymbol{V}}_{2}\right) .
\end{aligned}
$$

This field can be expressed in terms of degrees of freedom at time $t$ for the incremental displacements using the following relations:

$$
\begin{aligned}
& { }^{t+\Delta t} \underline{\boldsymbol{x}}_{M}-{ }^{t} \underline{\boldsymbol{x}}_{M}=\underline{\boldsymbol{u}}_{M}=u \underline{\boldsymbol{e}}_{1}+v \underline{\boldsymbol{e}}_{2}+w \underline{\boldsymbol{e}}_{3}, \\
& { }^{t+\Delta t} a-{ }^{t} a={ }^{0} a \cdot \Delta_{a}, \\
& { }^{t+\Delta t} Q_{n}-{ }^{t} Q_{n}=q_{n}, \\
& { }^{t+\Delta t} Q_{1}-{ }^{t} Q_{1}=q_{1}, \\
& { }^{t+\Delta t} Q_{2}-{ }^{t} Q_{2}=q_{2}, \\
& { }^{t+\Delta t} C_{1}-{ }^{t} C_{1}=c_{1}, \\
& { }^{t+\Delta t} C_{2}-{ }^{t} C_{2}=c_{2} .
\end{aligned}
$$

Here (6-a) represents three translations in the global Cartesian coordinate system given by the unit vectors $\underline{\boldsymbol{e}}_{i}$, with $i=1,2,3$, (6-b) gives a thickness change and (6-c-g) define displacement increments. Note that, in Eq. (6-b), the incremental thickness change is normalized by the initial thickness to circumvent ill-conditioning.

For the shell element, two rotational degrees of freedom have been generally used to describe the rotation of a director vector. We, instead, adopt three rotational degrees of freedom at this point and use

$$
\begin{aligned}
& { }^{t+\Delta t} \underline{\boldsymbol{V}}_{n}-{ }^{t} \underline{\boldsymbol{V}}_{n} \cong\left(\frac{1}{2} \gamma \alpha+\beta\right){ }^{t} \underline{\boldsymbol{V}}_{1}+\left(\frac{1}{2} \beta \gamma-\alpha\right){ }^{t} \underline{\boldsymbol{V}}_{2}-\frac{1}{2}\left(\alpha^{2}+\beta^{2}\right)^{t} \underline{\boldsymbol{V}}_{n}, \\
& { }^{t+\Delta t} \underline{\boldsymbol{V}}_{1}-{ }^{t} \underline{\boldsymbol{V}}_{1} \cong\left(\frac{1}{2} \alpha \beta+\gamma\right){ }^{t} \underline{\boldsymbol{V}}_{2}+\left(\frac{1}{2} \gamma \alpha-\beta\right) \underline{\boldsymbol{V}}_{n}-\frac{1}{2}\left(\beta^{2}+\gamma^{2}\right)^{t} \underline{\boldsymbol{V}}_{1}, \\
& { }^{t+\Delta t} \underline{\boldsymbol{V}}_{2}-{ }^{t} \underline{\boldsymbol{V}}_{2} \cong\left(\frac{1}{2} \beta \gamma+\alpha\right){ }^{t} \underline{\boldsymbol{V}}_{n}+\left(\frac{1}{2} \alpha \beta-\gamma\right){ }^{t} \underline{\boldsymbol{V}}_{1}-\frac{1}{2}\left(\gamma^{2}+\alpha^{2}\right)^{t} \underline{\boldsymbol{V}}_{2},
\end{aligned}
$$

where $\alpha, \beta, \gamma$ are the incremental rotations about the vectors ${ }^{t} \underline{\boldsymbol{V}}_{1},{ }^{t} \underline{\boldsymbol{V}}_{2}$, ${ }^{t} \underline{V}_{n}$, respectively. Once we have obtained $\alpha, \beta, \gamma$, the director vectors are updated with the following relationship:

${ }^{t+\Delta t} \underline{\boldsymbol{V}}_{\mathrm{i}}=\underline{\underline{\mathbf{Q}}} \underline{\boldsymbol{V}}_{\mathrm{i}} \quad(i=1,2, n)$,

\begin{tabular}{|c|c|c|c|c|}
\hline \multirow[b]{2}{*}{$\underline{u}_{\mathrm{L}}$} & \multicolumn{3}{|c|}{$\underline{\boldsymbol{u}}_{M}$} & A \\
\hline & $\frac{\xi}{2}$ & ${ }^{t} \underline{\mathbf{V}}_{1}$ & $\left({ }^{t} a\right) \beta$ & B \\
\hline & & ${ }^{t} \underline{\mathbf{V}}_{2}$ & $-\left({ }^{t} a\right) \alpha$ & C \\
\hline & & ${ }^{t} \underline{\mathbf{V}}_{n}$ & $\left({ }^{0} a\right) \Delta_{a}$ & D \\
\hline & $\xi^{20} a$ & ${ }^{t} \underline{\mathbf{V}}_{1}$ & ${ }^{t} Q_{n} \beta-{ }^{t} Q_{2} \gamma+q_{1}$ & E \\
\hline & & ${ }^{t} \underline{\mathbf{V}}_{2}$ & $-{ }^{t} Q_{n} \alpha+{ }^{t} Q_{1} \gamma+q_{2}$ & $\mathrm{~F}$ \\
\hline & & ${ }^{t} \underline{\mathbf{V}}_{n}$ & $-{ }^{t} Q_{1} \beta+{ }^{t} Q_{2} \alpha+q_{n}$ & G \\
\hline & $\xi^{30} a$ & ${ }^{t} \underline{\mathbf{V}}_{1}$ & $-{ }^{t} C_{2} \gamma+c_{1}$ & $\mathrm{H}$ \\
\hline & & ${ }^{t} \underline{\mathbf{V}}_{2}$ & ${ }^{t} C_{1} \gamma+c_{2}$ & I \\
\hline & & ${ }^{t} \underline{\mathbf{V}}_{n}$ & $-{ }^{t} C_{1} \beta+{ }^{t} C_{2} a$ & $\mathrm{~J}$ \\
\hline
\end{tabular}

where

$\underline{\underline{\boldsymbol{Q}}}=\underline{\underline{\boldsymbol{I}}}+\frac{\sin \theta}{\theta} \underline{\underline{\boldsymbol{S}}}+\frac{1}{2}\left(\frac{\sin (\theta / 2)}{\theta / 2}\right)^{2} \underline{\underline{\boldsymbol{S}}}^{2}$,

$\theta=\sqrt{\alpha^{2}+\beta^{2}+\gamma^{2}}$

$\underline{\underline{\boldsymbol{S}}}=\left[\begin{array}{ccc}0 & -\gamma & \beta \\ \gamma & 0 & -\alpha \\ -\beta & \alpha & 0\end{array}\right]$.

Note that Eqs. (7-a-c) are obtained from Eqs. (8) and (9). Using Eqs. (6) and (7) with Eq. (5), the incremental displacement field becomes $\underline{\boldsymbol{u}}=\underline{\boldsymbol{u}}_{\mathrm{L}}+\underline{\boldsymbol{u}}_{\mathrm{Q}}$,

where $\underline{\boldsymbol{u}}_{\mathrm{L}}$ and $\underline{\boldsymbol{u}}_{\mathrm{Q}}$ denote the linear and quadratic terms of incremental displacements, respectively,

$$
\begin{aligned}
& \underline{\boldsymbol{u}}_{\mathrm{L}}=\underline{\boldsymbol{u}}_{M}+\frac{\xi}{2}\left[{ }^{0} a^{t} \underline{\boldsymbol{V}}_{n} \Delta_{a}-{ }^{t} a^{t} \underline{\boldsymbol{V}}_{2} \alpha+{ }^{t} a^{t} \underline{\boldsymbol{V}}_{1} \beta\right] \\
& +\xi^{2}{ }^{0} a\left[\underline{\boldsymbol{V}}_{n} q_{n}-{ }^{t} Q_{n}{ }^{t} \underline{\boldsymbol{V}}_{2} \alpha+{ }^{t} Q_{n}{ }^{t} \underline{\boldsymbol{V}}_{1} \beta\right] \\
& +\xi^{2}{ }^{0} a\left[{ }^{t} \underline{\boldsymbol{V}}_{1} q_{1}-{ }^{t} Q_{1}{ }^{t} \underline{\boldsymbol{V}}_{n} \beta+{ }^{t} Q_{1}{ }^{t} \underline{\boldsymbol{V}}_{2} \gamma\right] \\
& +\xi^{2}{ }^{0} a\left[\underline{\boldsymbol{V}}_{2} q_{2}-{ }^{t} Q_{2}{ }^{t} \underline{\boldsymbol{V}}_{1} \gamma+{ }^{t} Q_{2}{ }^{t} \underline{\boldsymbol{V}}_{n} \alpha\right] \\
& +\xi^{3}{ }^{0} a\left[\underline{\boldsymbol{V}}_{1} c_{1}-{ }^{t} C_{1}{ }^{t} \underline{\boldsymbol{V}}_{n} \beta+{ }^{t} C_{1}{ }^{t} \underline{\boldsymbol{V}}_{2} \gamma\right] \\
& +\xi^{3}{ }^{0} a\left[\underline{\boldsymbol{V}}_{2} c_{2}-{ }^{t} C_{2}{ }^{t} \underline{\boldsymbol{V}}_{1} \gamma+{ }^{t} C_{2}{ }^{t} \underline{\boldsymbol{V}}_{n} \alpha\right] \\
& \underline{\boldsymbol{u}}_{\mathrm{Q}}=\frac{\xi}{2}\left[-{ }^{0} a^{t} \underline{\boldsymbol{V}}_{2} \Delta_{a} \alpha+{ }^{0} a^{t} \underline{\boldsymbol{V}}_{1} \Delta_{a} \beta+\frac{1}{2}{ }^{t} a^{t} \underline{\boldsymbol{V}}_{2} \beta \gamma+\frac{1}{2}{ }^{t} a^{t} \underline{\boldsymbol{V}}_{1} \gamma \alpha\right. \\
& \left.-\frac{1}{2}{ }^{t} a^{t} \underline{\boldsymbol{V}}_{n}\left(\alpha^{2}+\beta^{2}\right)\right]+\xi^{20} a\left[-{ }^{t} \underline{\boldsymbol{V}}_{2} q_{n} \alpha+{ }^{t} \underline{\boldsymbol{V}}_{1} q_{n} \beta\right. \\
& \left.+\frac{1}{2}{ }^{t} Q_{n}{ }^{t} \underline{\boldsymbol{V}}_{2} \beta \gamma+\frac{1}{2}{ }^{t} Q_{n}{ }^{t} \underline{\boldsymbol{V}}_{1} \gamma \alpha-\frac{1}{2}{ }^{t} Q_{n}{ }^{t} \underline{\boldsymbol{V}}_{n}\left(\alpha^{2}+\beta^{2}\right)\right] \\
& +\xi^{20} a\left[-{ }^{t} \underline{\boldsymbol{V}}_{n} q_{1} \beta+{ }^{t} \underline{\boldsymbol{V}}_{2} q_{1} \gamma+\frac{1}{2}{ }^{t} Q_{1}{ }^{t} \underline{\boldsymbol{V}}_{n} \gamma \alpha+\frac{1}{2}{ }^{t} Q_{1}{ }^{t} \underline{\boldsymbol{V}}_{2} \alpha \beta\right. \\
& \left.-\frac{1}{2}{ }^{t} Q_{1}{ }^{t} \underline{\boldsymbol{V}}_{1}\left(\beta^{2}+\gamma^{2}\right)\right]+\xi^{20} a\left[-{ }^{t} \underline{\boldsymbol{V}}_{1} q_{2} \gamma+{ }^{t} \underline{\boldsymbol{V}}_{n} q_{2} \alpha\right. \\
& \left.+\frac{1}{2}{ }^{t} Q_{2}{ }^{t} \underline{\boldsymbol{V}}_{1} \alpha \beta+\frac{1}{2}{ }^{t} Q_{2}{ }^{t} \underline{\boldsymbol{V}}_{n} \beta \gamma-\frac{1}{2}{ }^{t} Q_{2}{ }^{t} \underline{\boldsymbol{V}}_{2}\left(\gamma^{2}+\alpha^{2}\right)\right] \\
& +\xi^{3}{ }^{0} a\left[-{ }^{t} \underline{\boldsymbol{V}}_{n} c_{1} \beta+{ }^{t} \underline{\boldsymbol{V}}_{2} c_{1} \gamma+\frac{1}{2}{ }^{t} C_{1}{ }^{t} \underline{\boldsymbol{V}}_{n} \gamma \alpha+\frac{1}{2}{ }^{t} C_{1}{ }^{t} \underline{\boldsymbol{V}}_{2} \alpha \beta\right. \\
& \left.-\frac{1}{2}{ }^{t} C_{1}{ }^{t} \underline{\boldsymbol{V}}_{1}\left(\beta^{2}+\gamma^{2}\right)\right]+\xi^{30} a\left[-{ }^{t} \underline{\boldsymbol{V}}_{1} c_{2} \gamma+{ }^{t} \underline{\boldsymbol{V}}_{n} c_{2} \alpha\right. \\
& \left.+\frac{1}{2}{ }^{t} C_{2}{ }^{t} \underline{\boldsymbol{V}}_{1} \alpha \beta+\frac{1}{2}{ }^{t} C_{2}{ }^{t} \underline{\boldsymbol{V}}_{n} \beta \gamma-\frac{1}{2}{ }^{t} C_{2}{ }^{t} \underline{\boldsymbol{V}}_{2}\left(\gamma^{2}+\alpha^{2}\right)\right] \text {. }
\end{aligned}
$$

Table 1

The components of the linear incremental displacement given in Eq. (11) 
Table 2

Summary of shell models

\begin{tabular}{llll}
\hline Shell model & Nodal DOFs & $\begin{array}{l}\text { Constitutive } \\
\text { law }\end{array}$ & $\begin{array}{l}\text { Gauss integration } \\
\text { points }(r \times s \times \xi)\end{array}$ \\
\hline 5 DOF & $u, v, w, \alpha, \beta$ & Modified & $2 \times 2 \times 2$ \\
7 DOF & $u, v, w, \alpha, \beta$ & Full 3D & $2 \times 2 \times 2$ \\
\multirow{2}{*}{9 DOF } & $\Delta_{a}, q_{n}$ & Full 3D & $2 \times 2 \times 3$ \\
& $u, v, w, \alpha, \beta$ & & \\
11 DOF & $\Delta_{a}, q_{n}, q_{1}, q_{2}$ & Full 3D & $2 \times 2 \times 4$ \\
& $u, v, w, \alpha, \beta$ & & \\
& $\Delta_{a}, q_{n}, q_{1}, q_{2}, c_{1}, c_{2}$ & & \\
\hline
\end{tabular}

The current shell model contains 12 parameters to describe the shell behavior: three translations, three rotations, one thickness change, three quadratic displacements and two cubic displacements. However, we now exclude the $\gamma$ rotation effect in the above equations because the degree of freedom leads to spurious zero energy modes, even when the initially straight fiber transverse to the shell mid-surface has become curved. Consider the linear terms of the incremental displacements, $\underline{\boldsymbol{u}}_{\mathrm{L}}$, as arranged in Table 1 . We see that the displacement corresponding to the $\gamma$ rotation when a fiber is not straight can be described by the degrees of freedom $q_{1}, q_{2}, c_{1}$, $c_{2}$. Therefore, the $\gamma$ rotation is a redundant degree of freedom and can be set to zero when we use the higher-order in-plane displacements.

\section{Finite element discretization}

We use the shell model given in Section 2 for the finite element discretization.

\subsection{Interpolation of geometry and displacement}

To interpolate the shell geometry, the usual interpolation is used [1]

$$
\begin{aligned}
{ }^{t} \underline{\boldsymbol{x}}(r, s, \xi)= & \sum_{k=1}^{4} h^{k}(r, s)^{t} \underline{\boldsymbol{x}}_{M}^{k}+\frac{\xi}{2} \sum_{k=1}^{4} h^{k}(r, s)^{t} a^{k t} \underline{\boldsymbol{V}}_{n}^{k} \\
& +\xi^{2} \sum_{k=1}^{4} h^{k}(r, s){ }^{0} a^{k t} Q_{n}^{k t} \underline{\boldsymbol{V}}_{n}^{k} \\
& +\xi^{2} \sum_{k=1}^{4} h^{k}(r, s){ }^{0} a^{k}\left({ }^{t} Q_{1}^{k t} \underline{\boldsymbol{V}}_{1}^{k}+{ }^{t} Q_{2}^{k t} \underline{\boldsymbol{V}}_{2}^{k}\right) \\
& +\xi^{3} \sum_{k=1}^{4} h^{k}(r, s){ }^{0} a^{k}\left({ }^{t} C_{1}^{k}{ }^{t} \underline{\boldsymbol{V}}_{1}^{k}+{ }^{t} C_{2}^{k}{ }^{t} \underline{\boldsymbol{V}}_{2}^{k}\right),
\end{aligned}
$$

where $h^{k}(r, s)$ is the interpolation function corresponding to node $k$ (10)

Table 3

Cases of applied tractions and analytic stress distributions for the in-plane traction test

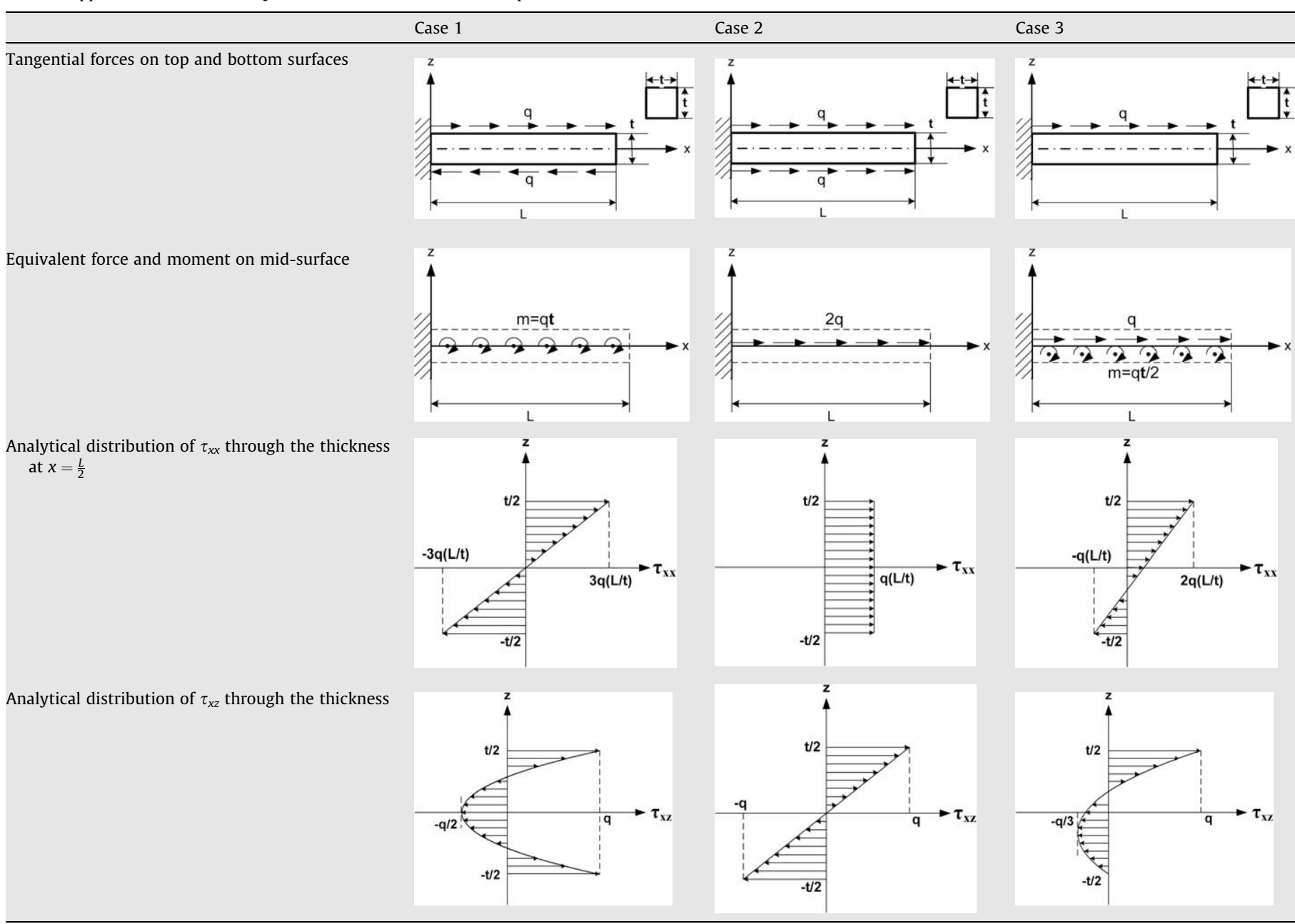

For simulations, stresses are evaluated at the center of the beam $\left(x=\frac{L}{2}\right)$ by using 41 elements along the beam with $L=20.0, t=1.0, q=10.0, E=1.0 \times 10^{5}, v=0.3$. 

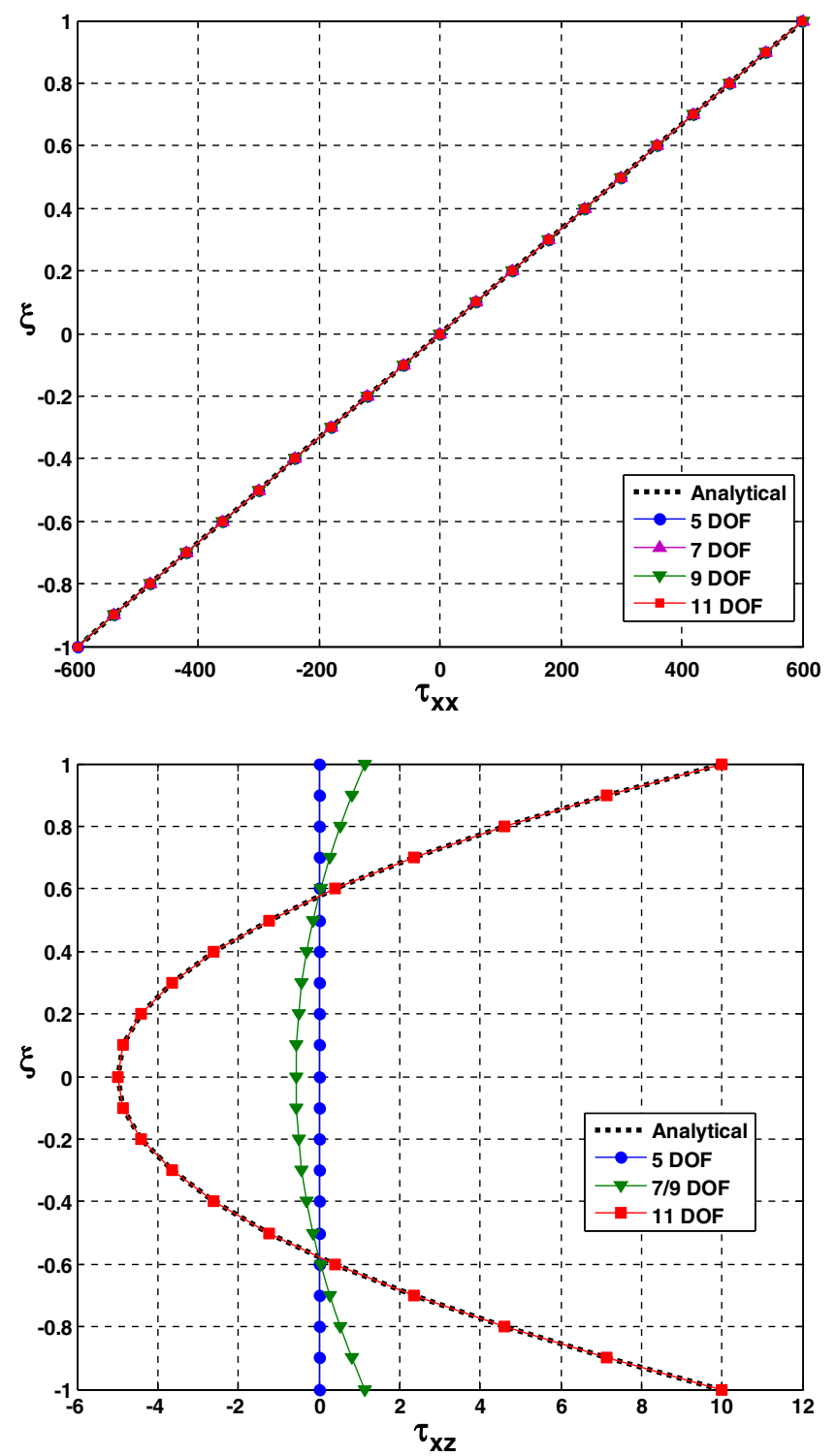

Fig. 3. Stress distributions in a cantilever beam under in-plane tractions (Case 1).

$\underline{\boldsymbol{u}}(r, s, \xi)=\sum_{k=1}^{4} h^{k}(r, s) \underline{\boldsymbol{u}}_{\mathrm{L}}^{k}+\sum_{k=1}^{4} h^{k}(r, s) \underline{\boldsymbol{u}}_{\mathrm{Q}}^{k}$.

\subsection{Mixed interpolation of strain field}

The total Lagrangian formulation is used for large deformation but small strain analysis. In this framework, the covariant components of the Green-Lagrange strain with respect to the initial configuration are defined by

${ }_{0}^{t} \tilde{\varepsilon}_{i j}=\frac{1}{2}\left(\underline{\boldsymbol{g}_{i}} \cdot{ }^{t} \underline{\mathbf{g}}_{j}-{ }^{0} \underline{\mathbf{g}}_{\mathrm{i}} \cdot{ }^{0} \underline{\mathbf{g}_{j}}\right)$,

where

${ }^{0} \underline{\boldsymbol{g}_{\mathrm{i}}}=\frac{\partial^{0} \underline{\boldsymbol{x}}}{\partial r_{\mathrm{i}}}, \quad{ }^{t} \underline{\boldsymbol{g}}_{\mathrm{i}}=\frac{\partial^{t} \underline{\boldsymbol{x}}}{\partial r_{\mathrm{i}}} \quad$ with $r_{1}=r, r_{2}=s, r_{3}=\xi$.

The incremental strains are directly calculated by

${ }_{0} \tilde{\varepsilon}_{i j}={ }_{0}^{t+\Delta t} \tilde{\varepsilon}_{i j}-{ }_{0}^{t} \tilde{\varepsilon}_{i j}=\frac{1}{2}\left(\frac{\partial \underline{\boldsymbol{u}}}{\partial r_{\mathrm{i}}} \cdot{ }^{t} \underline{\boldsymbol{g}}_{j}+\frac{\partial \underline{\boldsymbol{u}}}{\partial r_{j}} \cdot{ }^{t} \underline{\boldsymbol{g}}_{\mathrm{i}}+\frac{\partial \underline{\boldsymbol{u}}}{\partial r_{\mathrm{i}}} \cdot \frac{\partial \underline{\boldsymbol{u}}}{\partial r_{j}}\right)$.
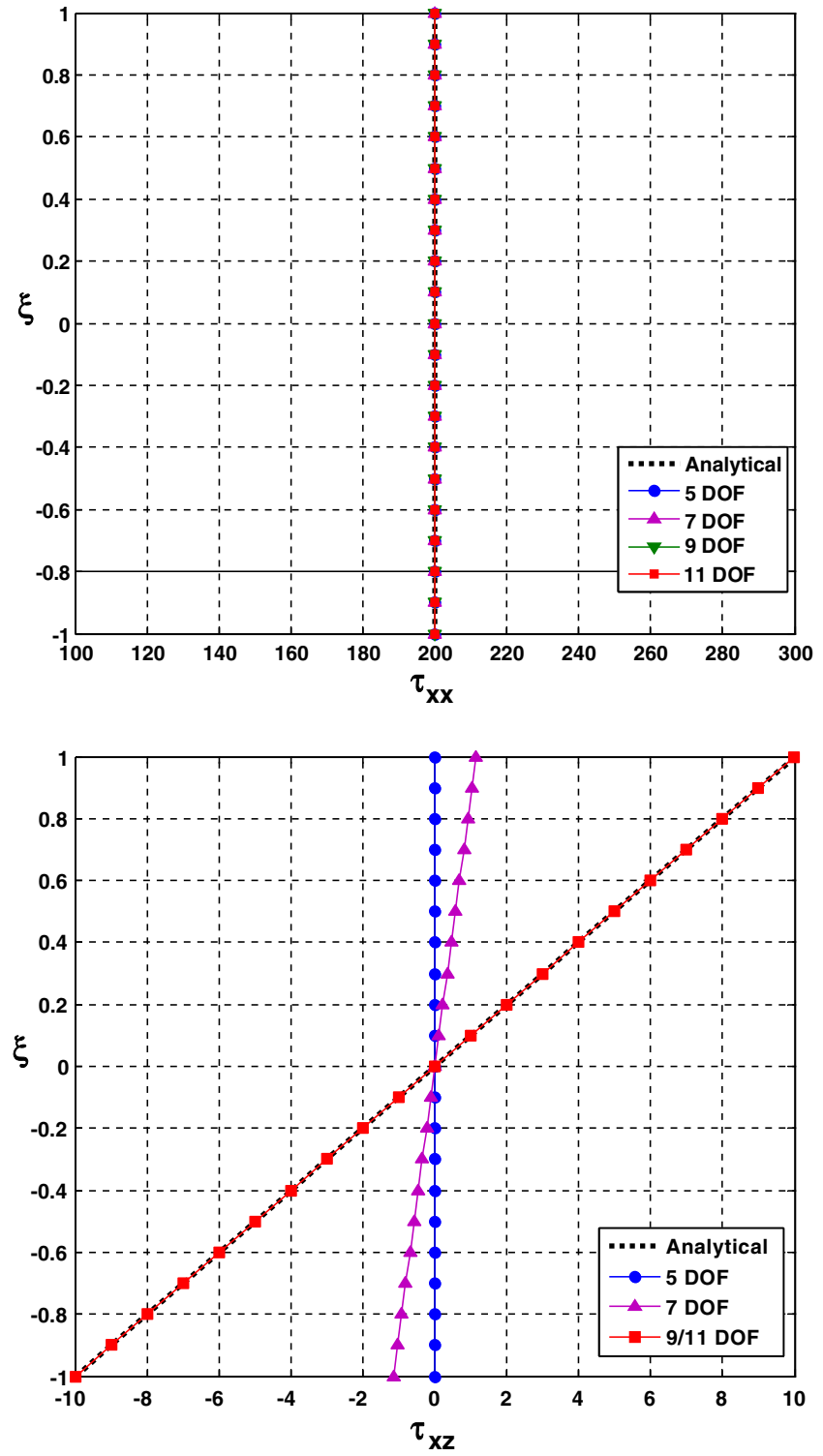

Fig. 4. Stress distributions in a cantilever beam under in-plane tractions (Case 2).

In order to avoid shear locking, the Dvorkin-Bathe transverse shear strain field is assumed, by interpolating the covariant components of the transverse shear strains using for all $\xi$

${ }_{0}^{t} \tilde{\varepsilon}_{13}^{\mathrm{AS}}=\left.\frac{1}{2}(1+s)_{0}^{t} \tilde{\varepsilon}_{13}^{\mathrm{DI}}\right|_{\substack{r=0 \\ s=1}}+\left.\frac{1}{2}(1-s)_{0}^{t} \tilde{\varepsilon}_{13}^{\mathrm{DI}}\right|_{\substack{r=0 \\ s=-1}}$,
${ }_{0}^{t} \tilde{\varepsilon}_{23}^{\mathrm{AS}}=\left.\frac{1}{2}(1+r)_{0}^{t} \tilde{\varepsilon}_{23}\right|_{\substack{r=1 \\ s=0}}+\left.\frac{1}{2}(1-r)_{0}^{t} \tilde{\varepsilon}_{23} \mathrm{DI}\right|_{\substack{r=-1 \\ s=0}}$.

Here the superscript AS denotes assumed strain and the superscript DI emphasizes that strains are evaluated from the displacement interpolation using Eq. (15).

While the basic MITC4 element does not show membrane locking, the in-plane membrane behavior can of course be improved by introducing incompatible displacement modes [1], as offered for example in ADINA [24]. A similar improvement in the membrane behavior must be expected when using incompatible displacement modes in the formulation of the enriched element presented here.

However, the strain assumptions normal to the shell mid-surface used here mean that the element will show pinching locking. 

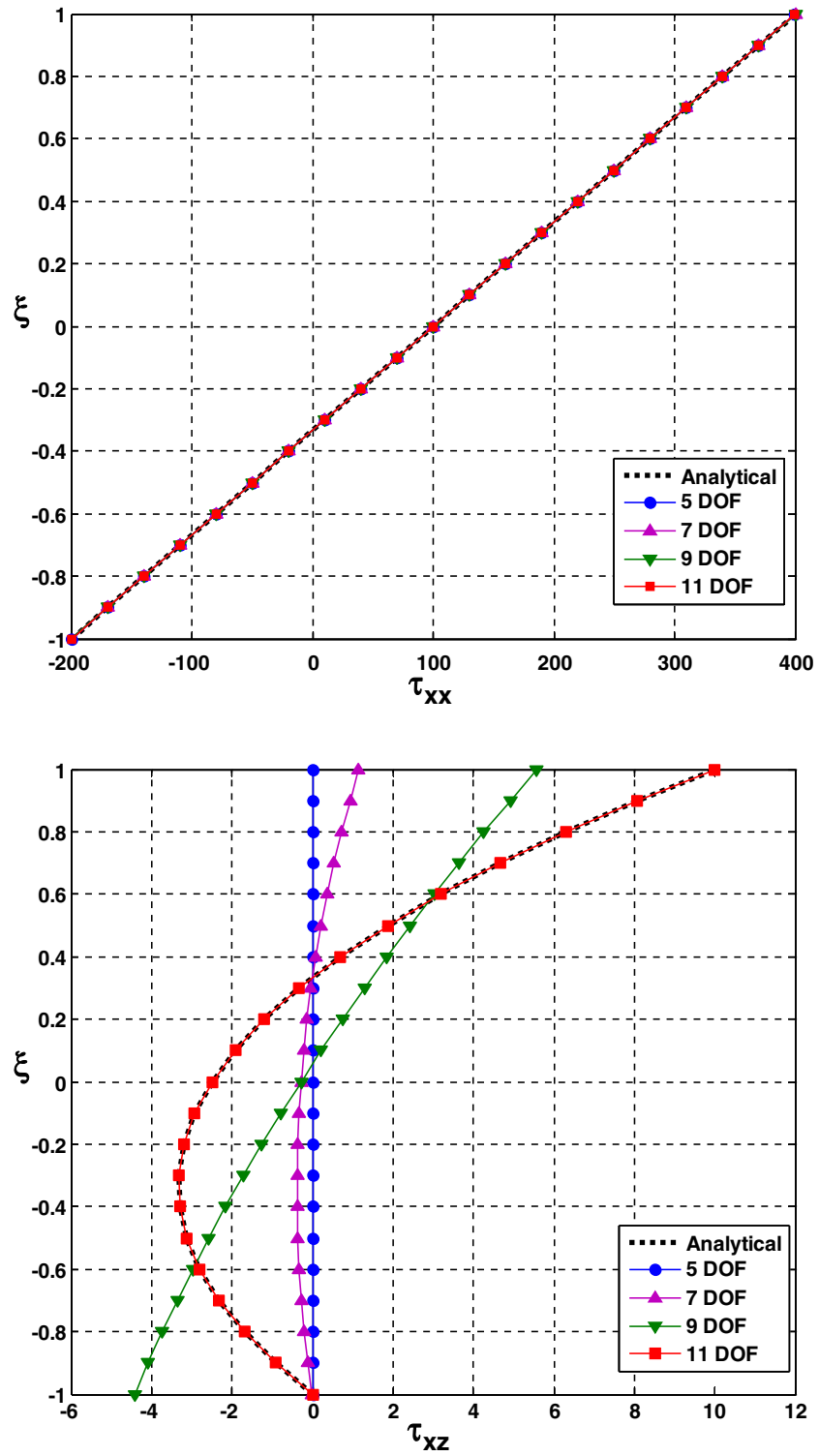

Fig. 5. Stress distributions in a cantilever beam under in-plane tractions (Case 3).
This behavior can be alleviated using the MITC approach applied to the normal strain $[12,25,26]$. To fulfill the condition that the normal strain be zero throughout the element when this strain is zero at the element nodes, we simply interpolate the normal strain bilinearly over the element using the nodal values directly calculated from the displacement assumptions. These nodal values will be zero for a constant bending situation even when the nodal director vectors are not normal to the shell mid-surface described by ${ }^{t} \underline{\boldsymbol{x}}_{M}$. Therefore, the assumed transverse normal strain is described by

${ }_{0}^{t} \tilde{\varepsilon}_{33}^{\mathrm{AS}}(r, s, \xi)=\left.{ }_{0}^{t} \tilde{\varepsilon}_{33}^{\mathrm{AS}}\right|_{\text {Const. }}+\left(\left.{ }_{0}^{t} \tilde{\varepsilon}_{33} \mathrm{DI}\right|_{r, s, \xi}-\left.{ }_{0}^{t} \tilde{\varepsilon}_{33} \mathrm{DI}\right|_{r, s, \xi=0}\right)$,

where

$$
\begin{aligned}
& \left.{ }_{0}^{t} \tilde{\varepsilon}_{33}^{\mathrm{AS}}\right|_{\text {Const. }}=\left.\frac{1}{4}(1+r)(1+s)_{0}^{t} \tilde{\varepsilon}_{33}^{\mathrm{DI}}\right|_{\substack{r=1 \\
s=1 \\
\xi=0}}+\left.\frac{1}{4}(1-r)(1+s)_{0}^{t} \widetilde{\varepsilon}_{33}^{\mathrm{DI}}\right|_{\substack{r=-1 \\
s=1 \\
\xi=0}} \\
& +\left.\frac{1}{4}(1-r)(1-s)_{0}^{t} \tilde{\varepsilon}_{33}^{\mathrm{DI}}\right|_{\substack{r=-1 \\
s=-1 \\
\xi=0}}+\left.\frac{1}{4}(1+r)(1-s)_{0}^{t} \tilde{\varepsilon}_{33}^{\mathrm{DI}}\right|_{\substack{r=1 \\
s=-1 \\
\xi=0}} .
\end{aligned}
$$

The behavior of this interpolation is thoroughly analyzed in Ref. [26]. All the other strain components are directly obtained using Eq. (15).

\subsection{Displacement/pressure ( $u / p)$ formulation}

Unlike shell elements based on the plane stress assumption, shell elements using the full three-dimensional constitutive law suffer also from volumetric locking in incompressible, (or almost incompressible) analysis, just like the elements used in the analysis of solids [1].

The mixed formulation known as the displacement/pressure $(u /$ p) formulation has been proven to be effective for the analysis of incompressible conditions [1,27]. Therefore, we adopt this formulation for the three-dimensional shell element. The key step of using the $u / p$ formulation is to determine the interpolation of the assumed independent pressure field. In the 4/1 plane strain element, the pressure field is assumed to be constant which means that strictly the element does not pass the inf-sup condition $[1,28]$. This is observed when regular meshes and special boundary conditions are used [1]. However, in practice, hardly flat and non-
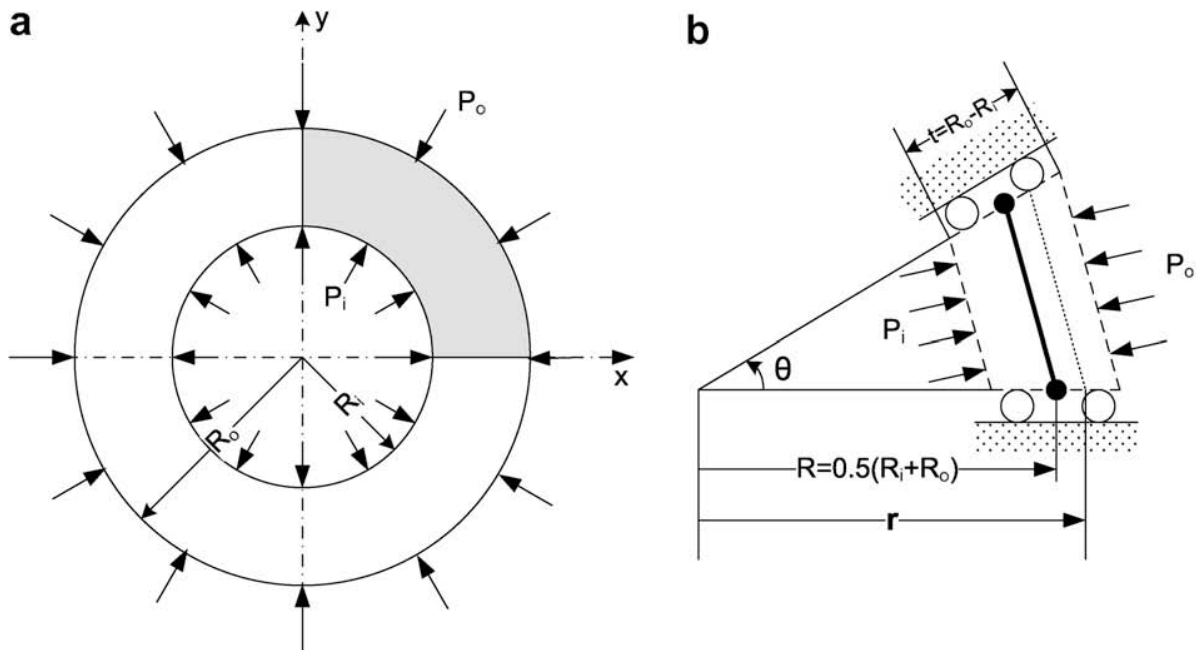

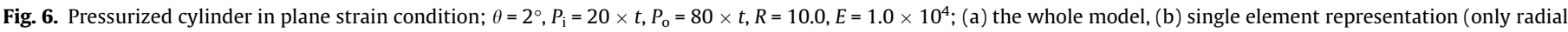
displacement is allowed). 
Table 4

Normalized radial displacement of the pressurized cylinder at $r=R$

\begin{tabular}{|c|c|c|c|c|c|c|}
\hline \multicolumn{3}{|c|}{ Thickness (t) } & \multirow{2}{*}{$\begin{array}{l}0.1 \\
0.9962\end{array}$} & \multirow{2}{*}{$\frac{1.0}{0.9643}$} & \multirow{2}{*}{$\begin{array}{l}2.0 \\
0.9294\end{array}$} & \multirow{2}{*}{$\frac{5.0}{0.8234}$} \\
\hline$\frac{u_{r}}{u_{r}}$ & $v=0.3$ & $5 \mathrm{DOF}$ & & & & \\
\hline & & $7 / 9 / 11 \mathrm{DOF}$ & 0.9998 & 0.9998 & 0.9998 & 0.9980 \\
\hline & $v=0.499999$ & 5 DOF & 0.9915 & 0.9203 & 0.8472 & 0.6495 \\
\hline & & 7/9/11 DOF & 0.9998 & 0.6150 & 0.0905 & 0.0025 \\
\hline & & $7 / 9 / 11$ DOF $(u / p)$ & 0.9998 & 0.9998 & 0.9998 & 0.9987 \\
\hline
\end{tabular}
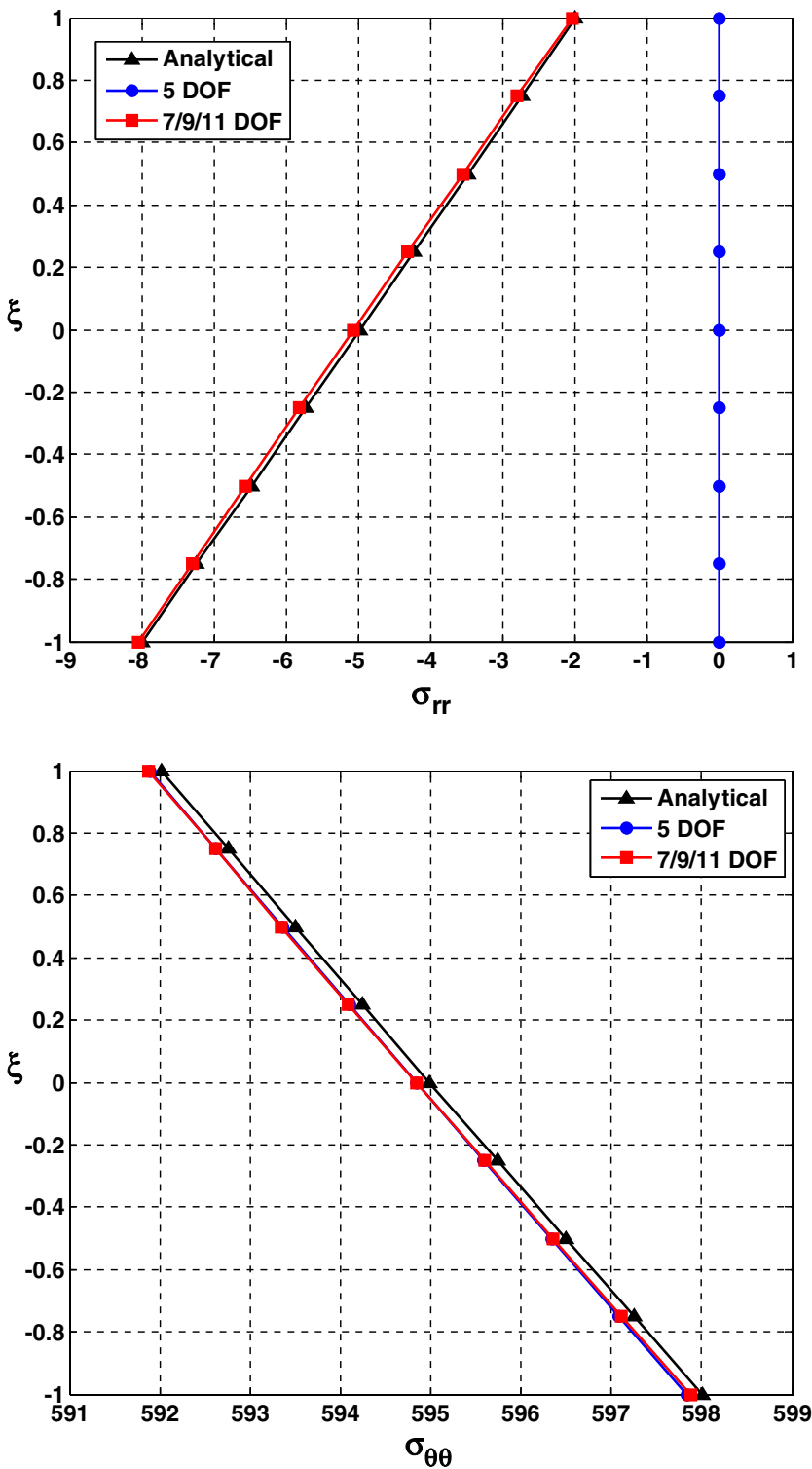

Fig. 7. Stress distributions in the pressurized cylinder when $t=0.1, P_{\mathrm{i}}=2.0, P_{\mathrm{o}}=8.0$.

distorted shell elements are employed, and therefore the following pressure variation is proposed for the element

$p=p_{0}+p_{1} \cdot \xi$.

Note that the pressure is assumed to be constant on each plane given by a fixed value of $\xi$ and vary linearly through the shell thickness. The linear pressure distribution is introduced considering bending. The cost increase by using this formulation is negligible since only two additional pressure degrees of freedom are added
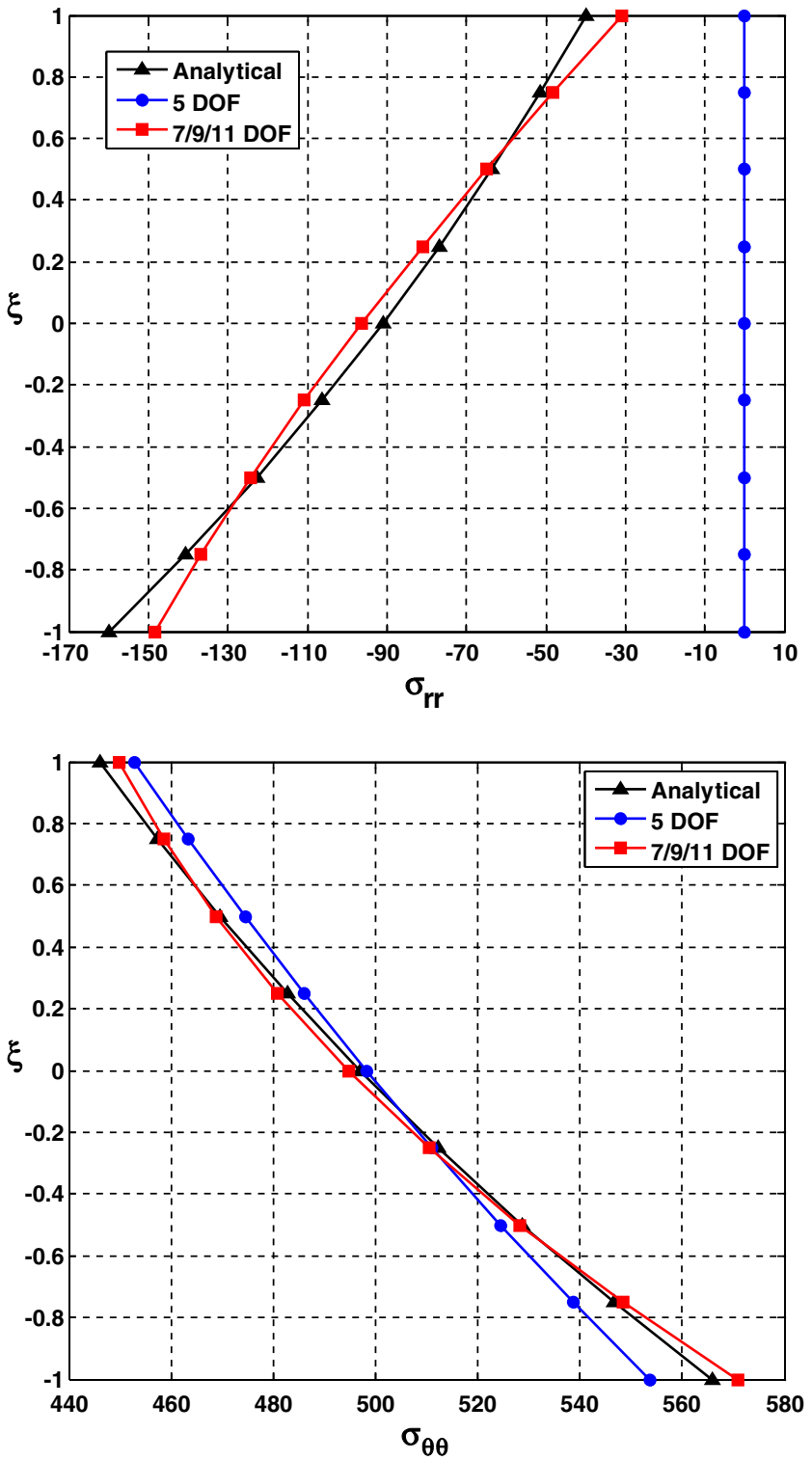

Fig. 8. Stress distributions in the pressurized cylinder when $t=2.0, P_{\mathrm{i}}=40, P_{\mathrm{o}}=160$.

to each element and these can be statically condensed out prior to the assemblage of the element stiffness matrix.

Since the 4/1 flat plane strain element (four nodes for displacements and a constant pressure) does not satisfy the inf-sup condition for incompressible analysis, there are very special element configurations and boundary conditions in which the shell element will also show checkerboard pressures. However, as mentioned already above, these will hardly be encountered in practice, and, also, can be identified in the post-processing of the results by plotting the pressure bands [1,31]. A 4-node plane strain element that satisfies the inf-sup condition is presented in Refs. [32,33]. The interpolation used in these references could be employed for the shell element but uses enhanced strains and would render the shell element in computations considerably more expensive.

\section{Numerical studies}

In this section, we illustrate some important features of our shell element through the results of several test problems. We 
a

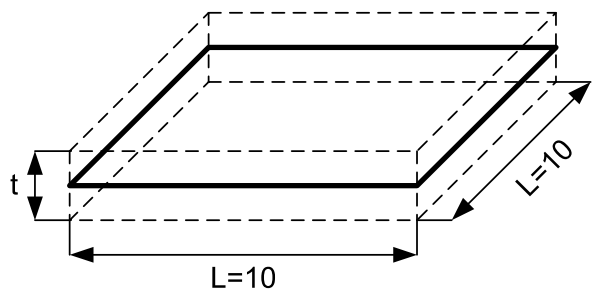

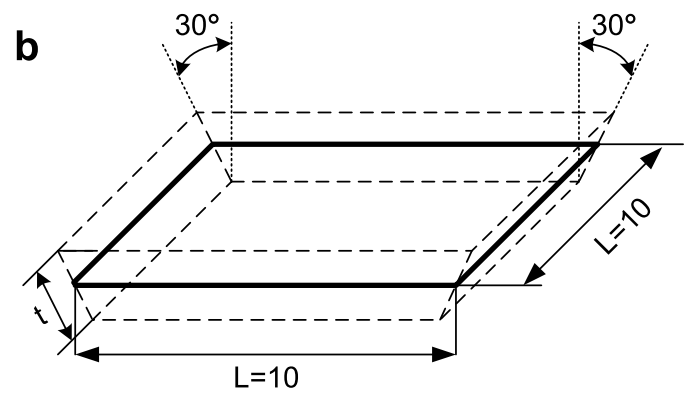

Fig. 9. Single element test for ill-conditioning: (a) director vectors are normal to the mid-surface, (b) director vectors are rotated $30^{\circ}$ from the normal direction.

Table 5

$C_{K}$ for different element thickness, see Fig. $9\left(E=1.0 \times 10^{7}, v=0.3\right)$

\begin{tabular}{llllrr}
\hline$t$ & & 1 & 0.1 & 0.01 & \multicolumn{1}{c}{0.001} \\
\hline 5 DOF & (a) & 3.8315 & 5.8217 & 7.8216 & 9.8216 \\
7 DOF & (b) & 4.0434 & 6.0365 & 8.0364 & 10.0364 \\
& (a) & 5.1716 & 7.1632 & 9.1632 & 11.1632 \\
$9 \mathrm{DOF}$ & (b) & 5.3384 & 7.3318 & 9.3318 & 11.3318 \\
& (a) & 5.1716 & 7.1632 & 9.1632 & 11.1632 \\
$11 \mathrm{DOF}$ & (b) & 5.4338 & 7.4268 & 9.4267 & 11.4267 \\
& (a) & 5.1732 & 7.1633 & 9.1632 & 11.1632 \\
3D 8-node Solid element (a) & 3.8963 & 7.8753 & 11.8750 & 15.5166
\end{tabular}

(a) director vectors are normal to the mid-surface, (b) director vectors are rotated $30^{\circ}$ from the normal direction.

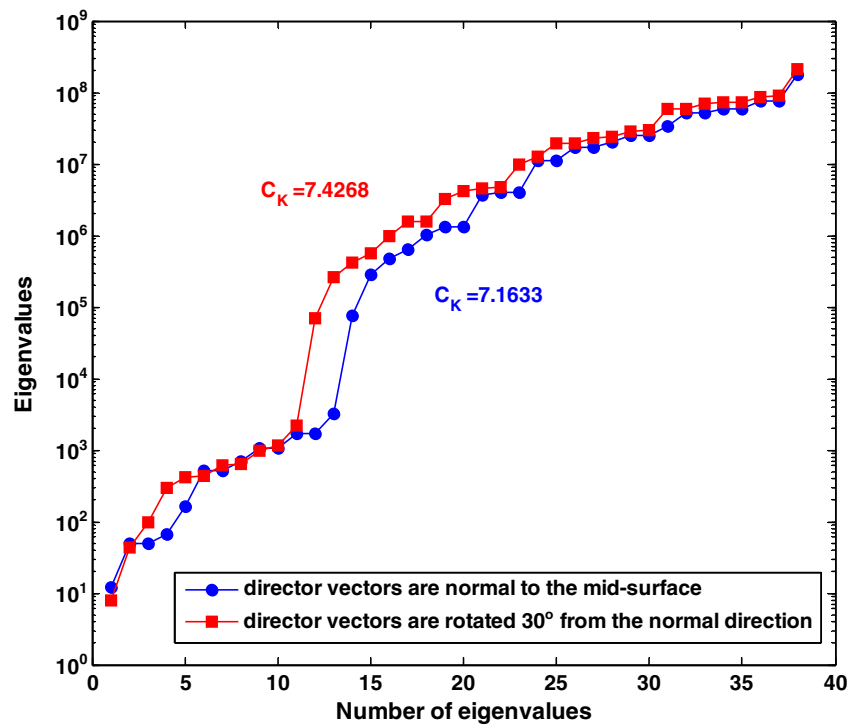

Fig. 10. Eigenvalues of single element (11-DOF, $E=1.0 \times 10^{7}, v=0.3, t=0.1$ ).

use our shell element with 5, 7, 9, or 11 degrees of freedom per node, see Table 2, which also lists the orders of numerical integration used. The element with 5 degrees of freedom at each node is the MITC4 shell element. Here, we use " $t$ " for the original shell thickness instead of ${ }^{0} a$ used above.

4.1. A cantilever beam under in-plane tangential tractions on the top and bottom surfaces

We consider a cantilever beam of rectangular cross-section with tangential in-plane tractions applied on its top and bottom surfaces. Of course, for normal tractions, the exact solution through the thickness is obtained (see also Section 4.2). In this problem,

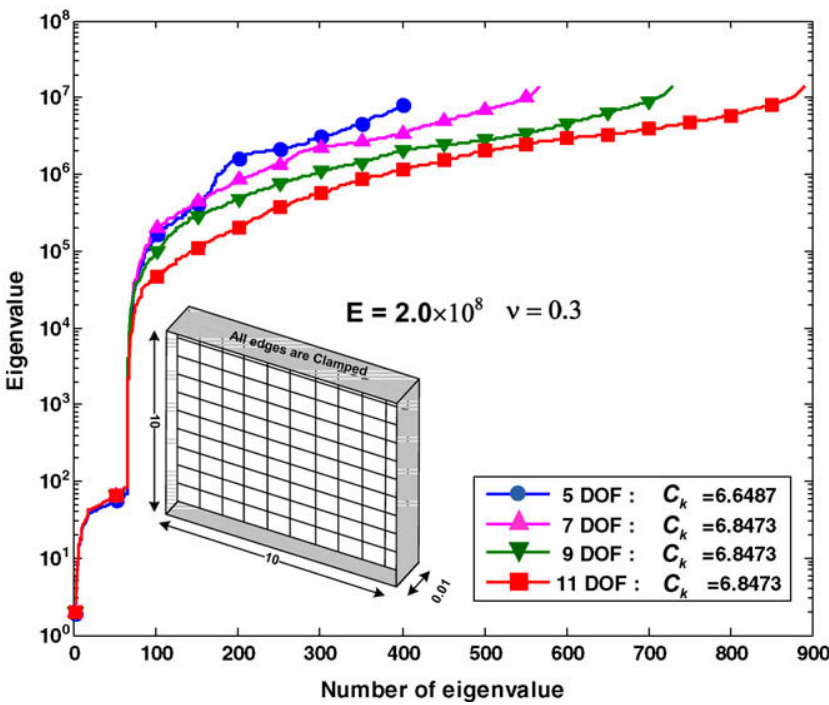

Fig. 11. Eigenvalues and condition numbers (director vectors normal to the midsurface).

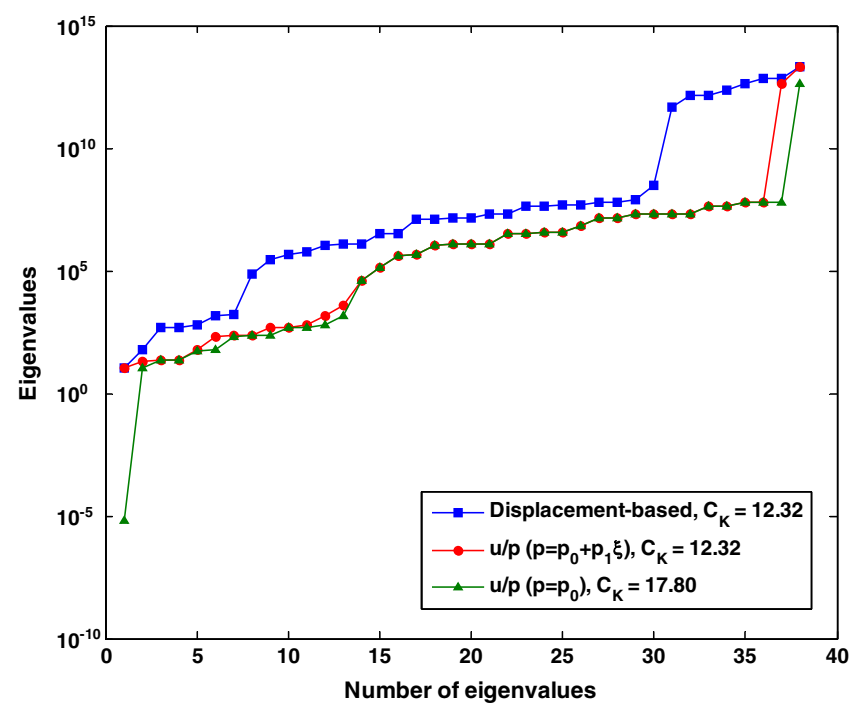

Fig. 12. Eigenvalues of single element in almost incompressible case (11-DOF element, $E=1.0 \times 10^{7}, v=0.499999, t=0.1$ ); director vectors normal to the midsurface.

the in-plane quadratic and cubic displacement functions corresponding to $q_{1}, q_{2}, c_{1}$ and $c_{2}$ play an important role in the prediction of the stress field. Three load cases are tested as shown in Table 3, 


\section{surface}
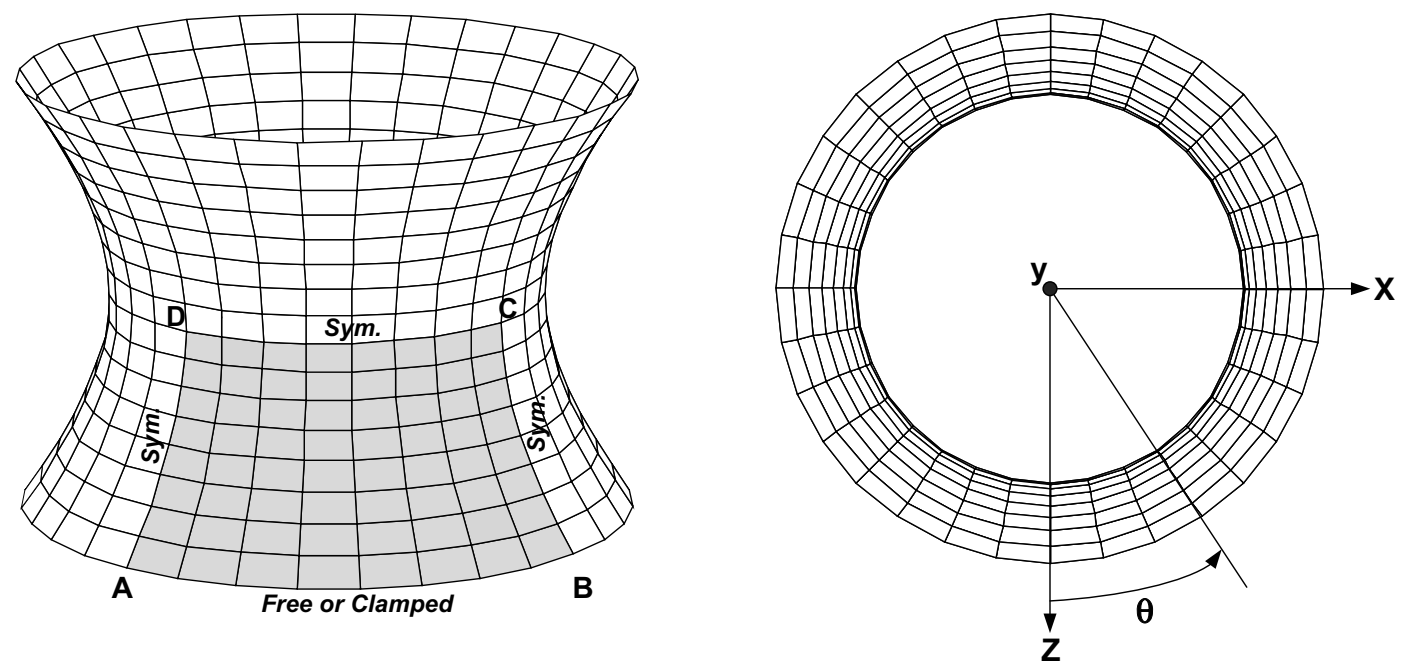

Fig. 13. The hyperboloid shell problem; $E=2.0 \times 10^{11} ; t=0.01 ; p_{\mathrm{o}}=1.0 \times 10^{6}$.

Table 6

Clamped-clamped boundary $(v=0.333333)$

\begin{tabular}{llll}
\hline & Strain energy & $\begin{array}{l}\text { Max. magnitude } \\
\text { of } V \text {-displacement }\end{array}$ & $\begin{array}{l}\text { Max. magnitude } \\
\text { of } U / W \text {-displacement }\end{array}$ \\
\hline 5 DOF & $5.33891 \mathrm{E}+02$ & $6.99667 \mathrm{E}-04$ & $1.35918 \mathrm{E}-03$ \\
$7 \mathrm{DOF}$ & $5.27460 \mathrm{E}+02$ & $7.01485 \mathrm{E}-04$ & $1.35438 \mathrm{E}-03$ \\
$9 \mathrm{DOF}$ & $5.28865 \mathrm{E}+02$ & $7.02342 \mathrm{E}-04$ & $1.35637 \mathrm{E}-03$ \\
$11 \mathrm{DOF}$ & $5.28915 \mathrm{E}+02$ & $7.02341 \mathrm{E}-04$ & $1.35635 \mathrm{E}-03$ \\
\hline
\end{tabular}

Table 7

Clamped-clamped boundary $(v=0.499999)$

\begin{tabular}{llll}
\hline & Strain energy & $\begin{array}{l}\text { Max. magnitude } \\
\text { of } V \text {-displacement }\end{array}$ & $\begin{array}{l}\text { Max. magnitude } \\
\text { of } U / W \text {-displacement }\end{array}$ \\
\hline $5 \mathrm{DOF}$ & $5.70978 \mathrm{E}+02$ & $7.56065 \mathrm{E}-04$ & $1.46331 \mathrm{E}-03$ \\
$7 \mathrm{DOF}$ & $0.40085 \mathrm{E}+02$ & $0.34764 \mathrm{E}-04$ & $0.18041 \mathrm{E}-03$ \\
$9 \mathrm{DOF}$ & $0.74792 \mathrm{E}+02$ & $0.57580 \mathrm{E}-04$ & $0.34114 \mathrm{E}-03$ \\
$11 \mathrm{DOF}$ & $1.18613 \mathrm{E}+02$ & $1.38555 \mathrm{E}-04$ & $0.36024 \mathrm{E}-03$ \\
$u / p-7$ DOF & $5.55900 \mathrm{E}+02$ & $7.59076 \mathrm{E}-04$ & $1.45204 \mathrm{E}-03$ \\
$u / p-9$ DOF & $5.58361 \mathrm{E}+02$ & $7.60885 \mathrm{E}-04$ & $1.45701 \mathrm{E}-03$ \\
$u / p-11$ DOF & $5.58467 \mathrm{E}+02$ & $7.60881 \mathrm{E}-04$ & $1.45706 \mathrm{E}-03$ \\
\hline
\end{tabular}

using $L=20.0, t=1.0, q=10.0, E=1.0 \times 10^{5}, v=0.3$. The beam is meshed with 41 elements along its length. We report the stresses as evaluated along the vertical centerline of the element located at the center of the beam, where edge effects are negligible. The analytical values for the stresses are derived in the Appendix.

In the first load case, the resultant axial force and shear force are zero but the bending moment is nonzero. As shown in Fig. 3, all shell element solutions give the same correct axial stress distribution. However, only the 11-DOF shell element predicts the correct shear stress distribution satisfying the zero resultant shear force condition and the traction boundary conditions on the top and bottom surfaces, $\tau_{x z \mid \xi}= \pm 1=q$.

In the second load case, the resultant shear force and bending moment are zero, but the axial force is nonzero. Fig. 4 shows the calculated stress distributions. In this case, both, the 9-DOF shell element and the 11-DOF shell element give the analytical solutions because the cubic displacement function has no effect on the finite element solution.
Table 8

Free-free boundary $(v=0.333333)$

\begin{tabular}{llll}
\hline & Strain energy & $\begin{array}{l}\text { Max. magnitude } \\
\text { of } V \text {-displacement }\end{array}$ & $\begin{array}{l}\text { Max. magnitude } \\
\text { of } U / W \text {-displacement }\end{array}$ \\
\hline 5 DOF & $4.53983 \mathrm{E}+05$ & $1.04082 \mathrm{E}+00$ & $2.10145 \mathrm{E}+00$ \\
$7 \mathrm{DOF}$ & $4.53820 \mathrm{E}+05$ & $1.04044 \mathrm{E}+00$ & $2.10070 \mathrm{E}+00$ \\
$9 \mathrm{DOF}$ & $4.55217 \mathrm{E}+05$ & $1.04372 \mathrm{E}+00$ & $2.10719 \mathrm{E}+00$ \\
$11 \mathrm{DOF}$ & $4.55237 \mathrm{E}+05$ & $1.04377 \mathrm{E}+00$ & $2.10728 \mathrm{E}+00$ \\
\hline
\end{tabular}

Table 9

Free-free boundary $(v=0.499999)$

\begin{tabular}{llll}
\hline & Strain energy & $\begin{array}{l}\text { Max. magnitude } \\
\text { of } V \text {-displacement }\end{array}$ & $\begin{array}{l}\text { Max. magnitude } \\
\text { of } U / W \text {-displacement }\end{array}$ \\
\hline 5 DOF & $3.81804 \mathrm{E}+05$ & $0.87391 \mathrm{E}+00$ & $1.76575 \mathrm{E}+00$ \\
$7 \mathrm{DOF}$ & $0.02886 \mathrm{E}+05$ & $0.00198 \mathrm{E}+00$ & $0.00846 \mathrm{E}+00$ \\
$9 \mathrm{DOF}$ & $1.45692 \mathrm{E}+05$ & $0.33107 \mathrm{E}+00$ & $0.66385 \mathrm{E}+00$ \\
$11 \mathrm{DOF}$ & $1.79671 \mathrm{E}+05$ & $0.40899 \mathrm{E}+00$ & $0.82209 \mathrm{E}+00$ \\
$u / p-7$ DOF & $3.81421 \mathrm{E}+05$ & $0.87293 \mathrm{E}+00$ & $1.76395 \mathrm{E}+00$ \\
$u / p-9$ DOF & $3.82368 \mathrm{E}+05$ & $0.87517 \mathrm{E}+00$ & $1.76836 \mathrm{E}+00$ \\
$u / p-11$ DOF & $3.82384 \mathrm{E}+05$ & $0.87520 \mathrm{E}+00$ & $1.76843 \mathrm{E}+00$ \\
\hline
\end{tabular}

In the third load case, the resultant axial force and bending moment are nonzero, but the resultant shear force is zero. Fig. 5 gives the calculated results. The predicted transverse shear stress is quite different using the various element assumptions. Only the 11-DOF shell element gives an accurate solution satisfying the traction boundary conditions on the top and bottom surfaces, $\tau_{x z \mid \xi=1}=q$, $\tau_{x z \mid \xi=-1}=0$. This result must be expected, since the case is a linear superposition of cases 1 and 2 .

Here, in general, the 11-DOF shell element with the quadratic distribution of the transverse shear stress through its thickness must be used to accurately capture the effect of tangential tractions on the top and bottom shell surfaces (satisfying the traction boundary conditions).

\subsection{Pressurized cylinder}

We consider the pressurized cylinder problem shown in Fig. 6. We use this problem to test the predictive capabilities 

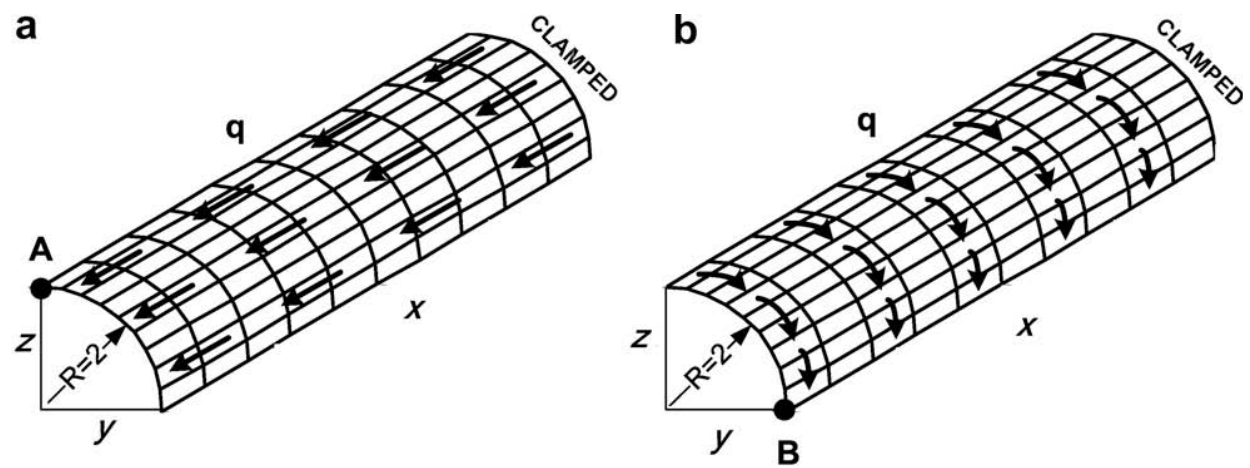

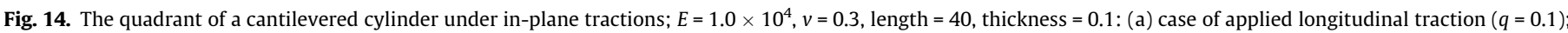
(b) case of applied circumferential traction $(q=0.007)$.

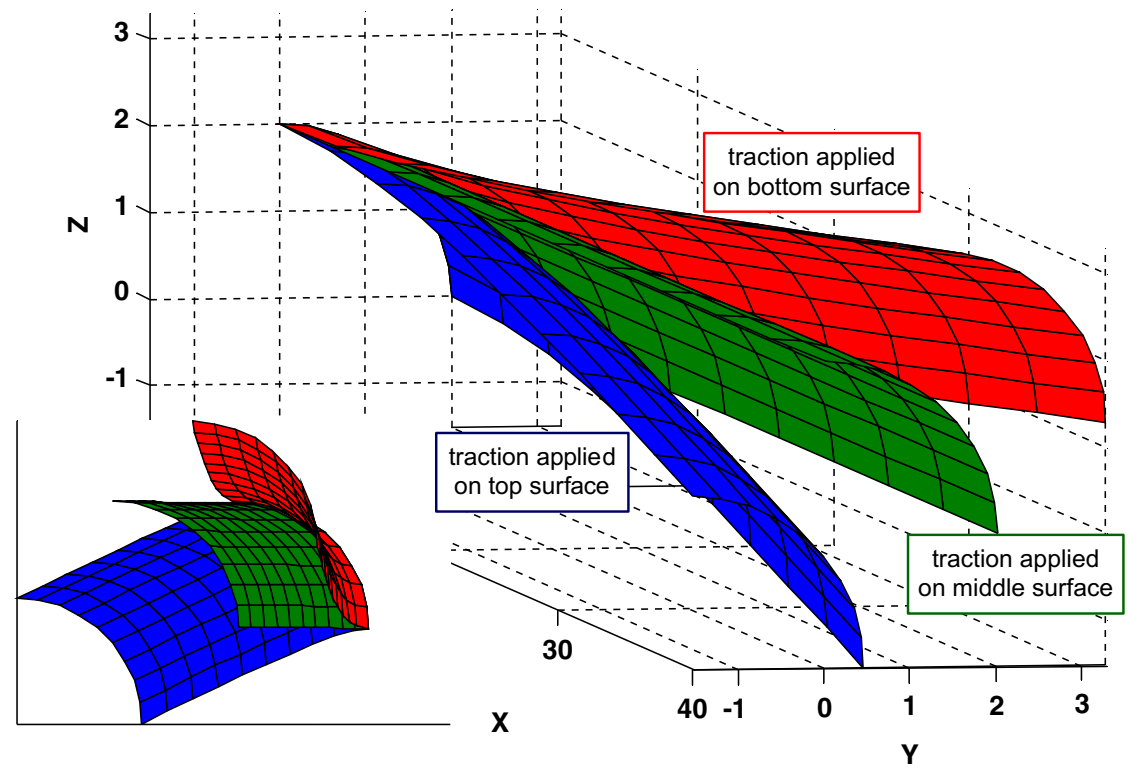

Fig. 15. The deformed shapes when the traction is applied in the longitudinal direction.

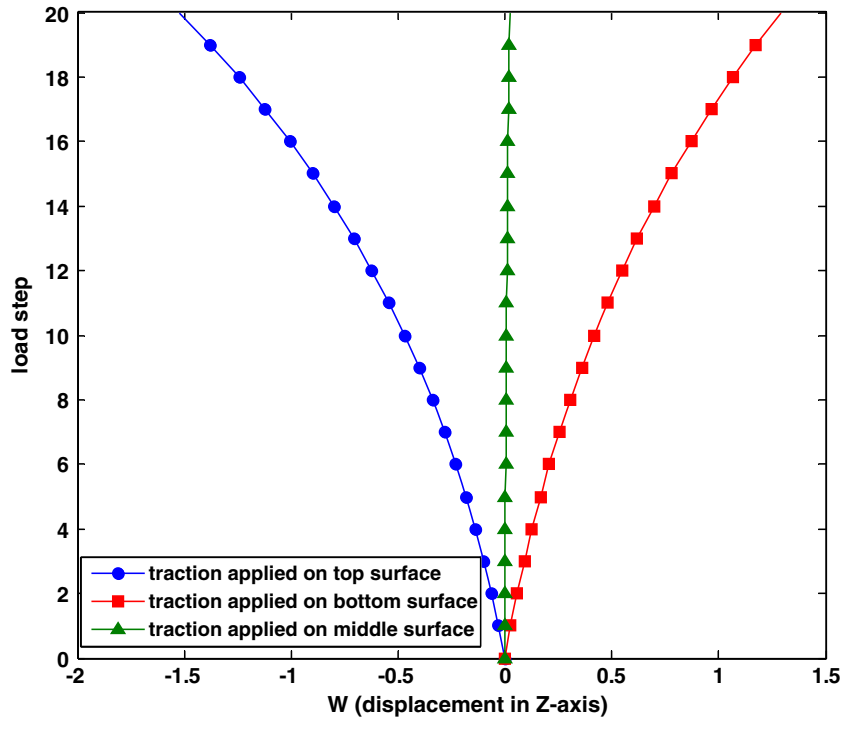

Fig. 16. Displacement in $z$-direction of point A in Fig. 14a. of our shell element in the analysis of a thick-walled structure. A one element model is used, see Fig. $6 \mathrm{~b}$. Note that, since the cylinder can only expand or contract in the radial direction due to symmetry, the degrees of freedom for the in-plane quadratic and cubic displacement functions $\left(q_{1}, q_{2}, c_{1}\right.$, and $\left.c_{2}\right)$ have no effect in the solution of this problem, i.e. the 9-DOF and 11-DOF shell elements will give the same result as the 7-DOF element.

The analytical solutions of this plane strain problem $\left(\varepsilon_{z z}=0\right)$ for the radial displacement $u_{r}$, and the radial and circumferential stress $\tau_{r r}$ and $\tau_{\theta \theta}$ are

$$
\begin{aligned}
u_{r} & =\frac{P_{\mathrm{i}} R_{\mathrm{i}}^{2}-P_{\mathrm{o}} R_{\mathrm{o}}^{2}}{2(\lambda+G)\left(R_{\mathrm{o}}^{2}-R_{\mathrm{i}}^{2}\right)} \times r+\frac{R_{\mathrm{i}}^{2} R_{\mathrm{o}}^{2}\left(P_{\mathrm{i}}-P_{\mathrm{o}}\right)}{2 G\left(R_{\mathrm{o}}^{2}-R_{\mathrm{i}}^{2}\right)} \times \frac{1}{r}, \\
\tau_{r r} & =\frac{P_{\mathrm{i}} R_{\mathrm{i}}^{2}-P_{\mathrm{o}} R_{\mathrm{o}}^{2}}{R_{\mathrm{o}}^{2}-R_{\mathrm{i}}^{2}}-\frac{R_{\mathrm{i}}^{2} R_{\mathrm{o}}^{2}\left(P_{\mathrm{i}}-P_{\mathrm{o}}\right)}{R_{\mathrm{o}}^{2}-R_{\mathrm{i}}^{2}} \times \frac{1}{r^{2}}, \\
\tau_{\theta \theta} & =\frac{P_{\mathrm{i}} R_{\mathrm{i}}^{2}-P_{\mathrm{o}} R_{\mathrm{o}}^{2}}{R_{\mathrm{o}}^{2}-R_{\mathrm{i}}^{2}}+\frac{R_{\mathrm{i}}^{2} R_{\mathrm{o}}^{2}\left(P_{\mathrm{i}}-P_{\mathrm{o}}\right)}{R_{\mathrm{o}}^{2}-R_{\mathrm{i}}^{2}} \times \frac{1}{r^{2}},
\end{aligned}
$$

where $R_{\mathrm{o}}$ and $R_{\mathrm{i}}$ are the outer and inner radii, respectively, $P_{\mathrm{o}}$ and $P_{\mathrm{i}}$ are the applied pressures, $\lambda$ and $G$ are the Lamé constants, and $R_{\mathrm{i}} \leqslant r \leqslant R_{\mathrm{o}}$ 


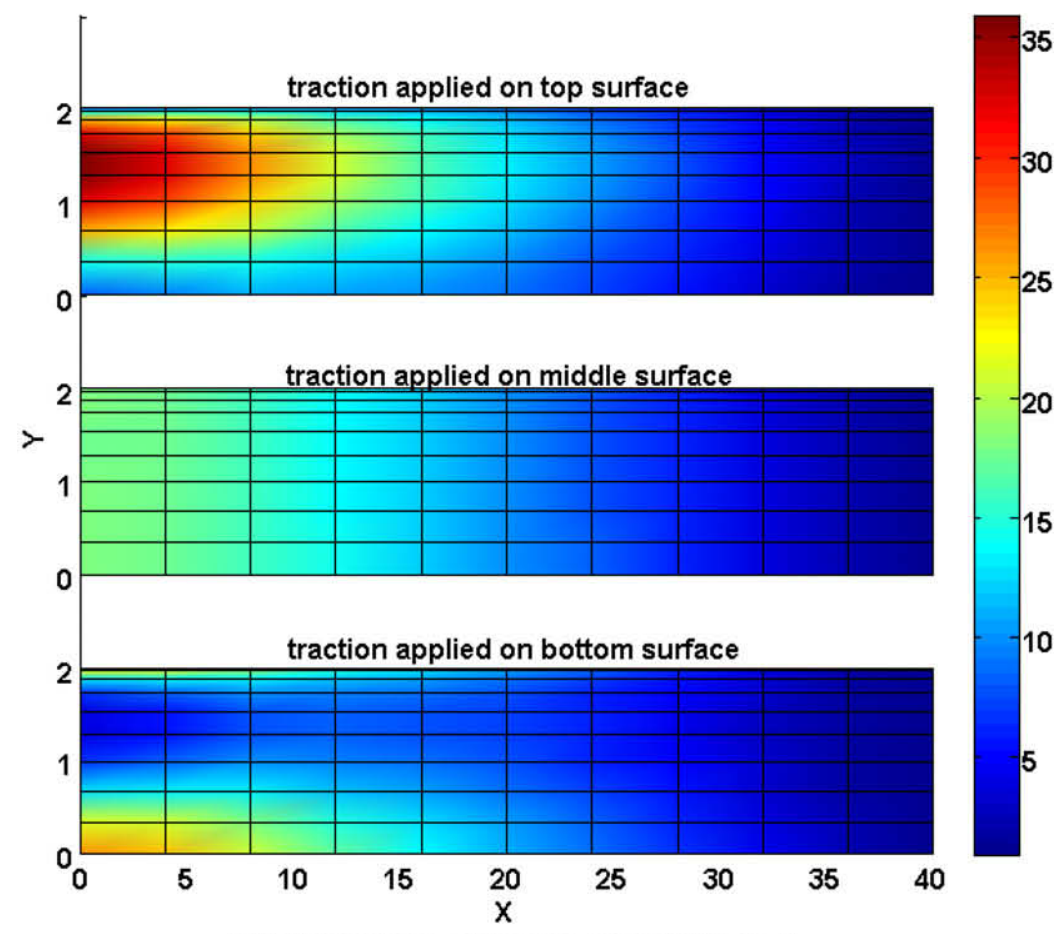

Effective Stress on the top surface (Case a)

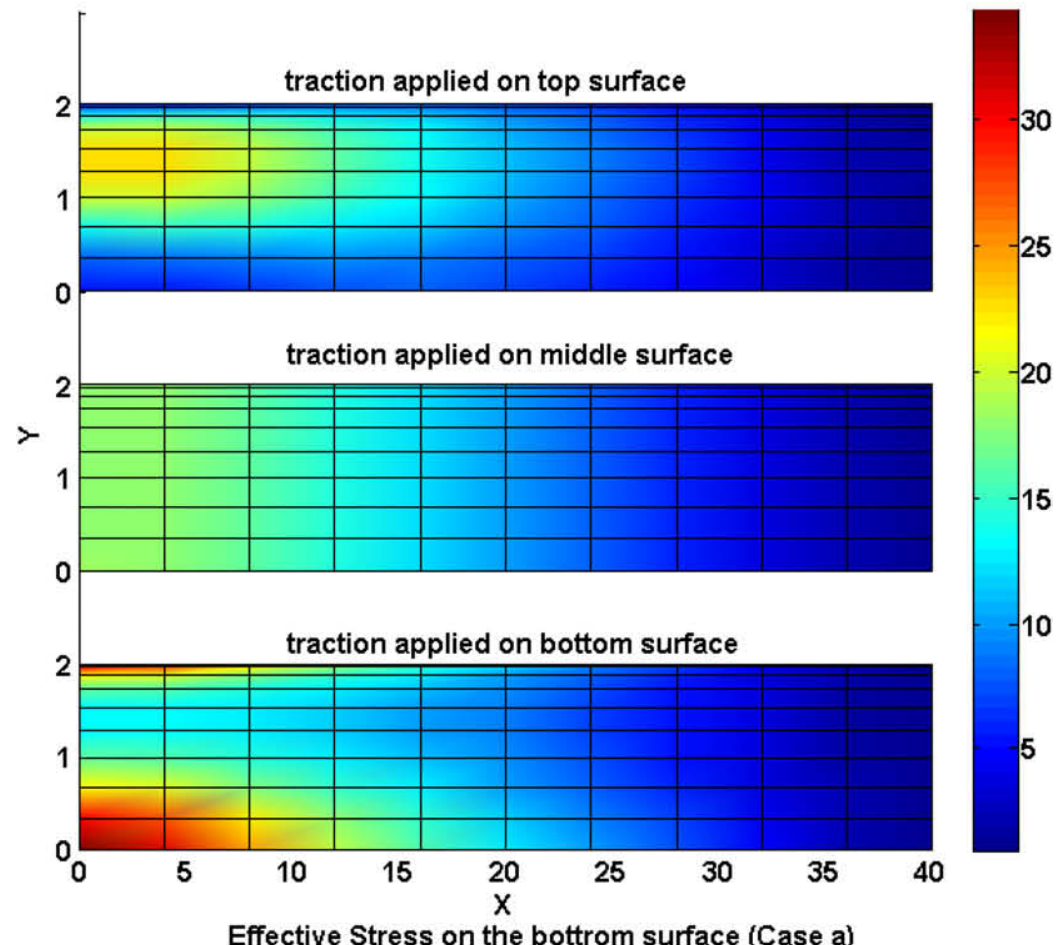

Fig. 17. The effective stress at the top and bottom surfaces in Case (a) of Fig. 14.

Table 4 lists the predicted radial displacement of the cylinder mid-surface, and shows that the displacement obtained using the 7-DOF shell element is in good agreement with the analytical solution, even when the cylinder is rather thick. However, in the (almost) incompressible case, the 7-DOF shell element needs to be used with the proposed assumed pressure field. Of course, if the cylinder is thin, both, the 5-DOF and 7-DOF shell elements give virtually the same result for the mid-surface displacement. Figs. 7 and
8 show the predicted radial and hoop stresses for the thin and thick cases.

\subsection{Test case for conditioning of the stiffness matrix}

In these problem solutions, we study the conditioning of the stiffness matrices when the thickness of the shell decreases and 
the material becomes incompressible. The degree of ill-conditioning can be measured by the condition number defined as [1],

$\operatorname{cond}(K)=\frac{\lambda_{\max }}{\lambda_{\min }}$

where $\lambda_{\max }$ and $\lambda_{\min }$ are the maximum and minimum eigenvalues of the stiffness matrix $K$ with rigid body modes excluded. For comparison purposes, we use $C_{K}$

$C_{K}=\log _{10}\{\operatorname{cond}(K)\}$.

First, a single element with two different orientations of director vectors as shown in Fig. 9 is tested. The results are listed in Table 5 . We see that the condition number of the 3D-shell element with more than five degrees of freedom is for the thin case only about an order of magnitude larger than for the MITC4 shell element with five degrees of freedom. This increase in the condition number is small when compared to the increase for the 3D solid element. Note that $\operatorname{cond}(K) \sim \mathcal{O}\left(t^{-2}\right)$ for all shell elements while cond $(K) \sim \mathcal{O}\left(t^{-4}\right)$ for the 3D solid element with the thickness, $t$. When the director vectors are not normal to the shell mid-surface as shown in Fig. 9b, the condition number only slightly increases. This is shown in Fig. 10.

Next, we consider a plate modeled with a $10 \times 10$ element mesh, see Fig. 11, and solve for the eigenvalues and condition numbers, see Ref. [17] for a similar example. We see that the condition numbers of our shell element with 7-11 degrees of freedom are only slightly larger than for the MITC4 element. Therefore, in the analysis of shell structures in which 3D effects shall be predicted, the element proposed here is much more reliable than the 3D solid element.

Finally, the single element with an almost incompressible material is considered. In this case, we have inevitably an ill-conditioned stiffness matrix because, for 3D type elements, we have at least one very large eigenvalue corresponding to the volumetric deformation mode. In Fig. 12, the displacement-based (without pressure degrees of freedom) shell element shows eight very large eigenvalues that cause the element to be too stiff and lock, see Tables 4,7 and 9 . If we use the element with the $\mathrm{u} / \mathrm{p}$ formulation, see the assumed pressure field in Eq. (21), only two very large eigenvalues which correspond to the volumetric expansion mode (the constant pressure mode) and a bending mode (the linear pressure mode through the thickness) are observed. It appears natural to therefore try to use only the constant pressure field $\left(p=p_{0}\right)$ in the $\mathrm{u} / \mathrm{p}$ formulation, but then the element has a spurious zero energy mode corresponding to the bending mode. Hence, the pressure assumption in Eq. (21) is more appropriate.

\subsection{Hyperboloid shell problems}

These shell problems were proposed and studied by Chapelle and Bathe $[2,29,30]$ and provide excellent test problems for shell elements. We solve the problems here to investigate the performance of our shell discretizations in the almost incompressible case.

The geometry of the shell mid-surface is given by (see Fig. 13)

$x^{2}+z^{2}=1+y^{2}, \quad y \in[-1,1]$.

The hyperboloid mid-surface is subjected to the smoothly varying periodic pressure

$p(\theta)=p_{0} \cos (2 \theta)$.

If the shell is clamped on both ends, the problem is a membranedominated problem, and if the shell is free on both ends, the problem is a bending-dominated problem. We use a $16 \times 16$ uniform mesh of elements to solve the two problems.

Some results are listed in Tables 6-9. When the material is compressible (see Tables 6 and 8 ), each shell model gives similar results in both the membrane and bending dominated cases. However, when the material is nearly incompressible (see Tables 7 and 9 ), the shell elements based on the full three-dimensional constitutive law experience volumetric locking while the 5-DOF MITC4 shell element of course does not lock. Note that, by using the $u / p$ formulation, the volumetric locking of the shell elements using the three dimensional constitutive law is successfully removed in, both, the membrane and bending dominated cases.

\subsection{A quadrant of a cantilevered cylinder under in-plane tangential tractions}

A quadrant of a cantilevered cylinder under in-plane tangential tractions is tested. The tractions are applied in the longitudinal direction for one case and the circumferential direction for the other case as depicted in Fig. 14. In order to see how the load positions affect the solutions, the deformation-dependent tractions are applied on the top, middle and bottom surfaces for each case.

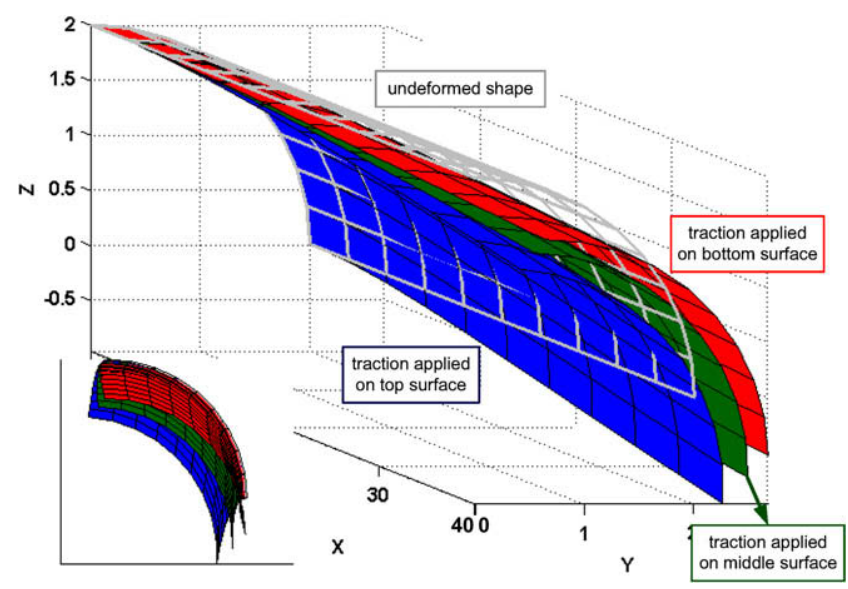

Fig. 18. The deformed shapes when the traction is applied in the circumferential direction.

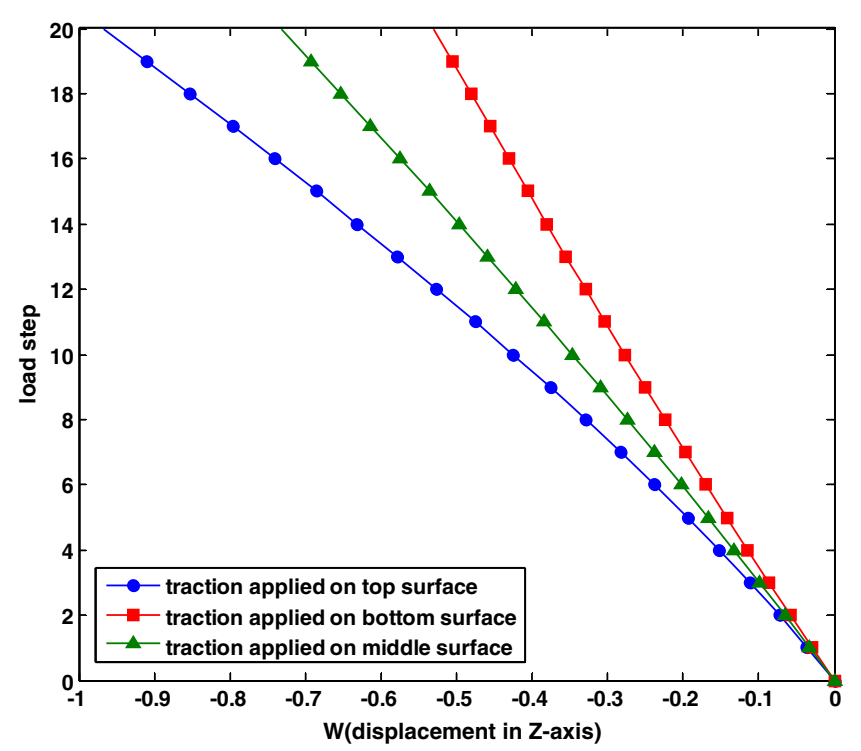

Fig. 19. Displacement in $z$-direction of point B in Fig. 14b. 


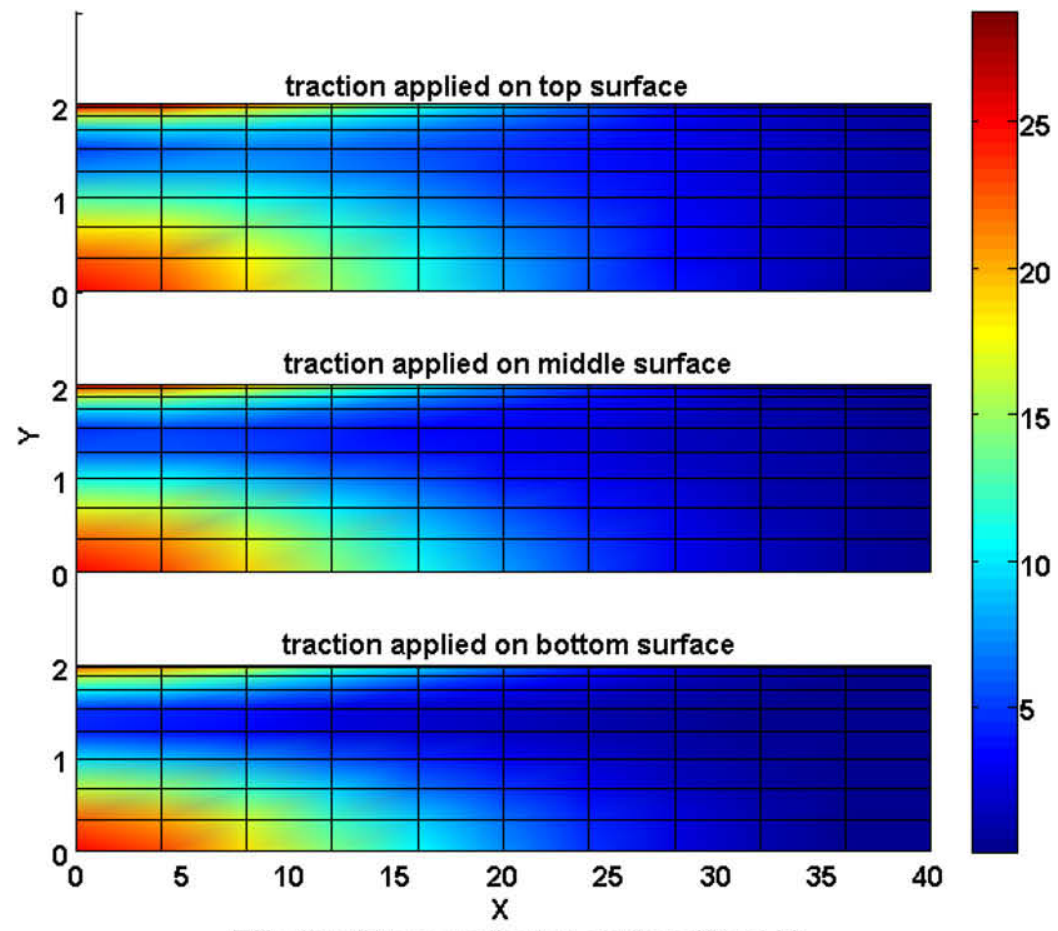

Effective Stress on the top surface (Case b)

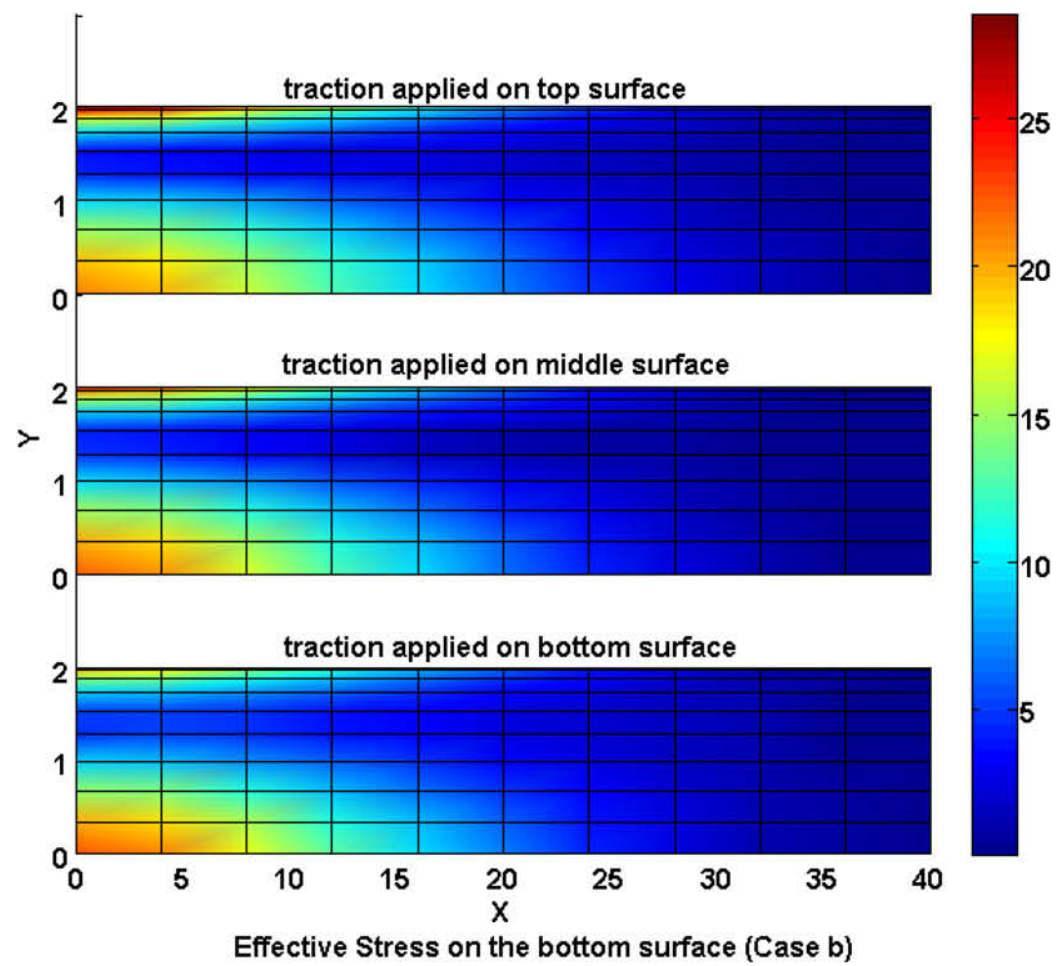

Fig. 20. The effective stress at the top and bottom surfaces in Case (b) of Fig. 14.

Geometrically nonlinear analyses are performed using the 11-DOF shell element with a $10 \times 9$ mesh of elements in the total Lagrangian framework [1]. The deformed shapes and the displacements and effective stresses are given in Figs. 15-20. We can see that significant response differences arise depending on how the tractions are applied.

Note that we may obtain very similar results with the 5-DOF MITC4 shell element by applying the resultant forces and moments on the shell mid-surface, especially for a very thin structure as in this problem. Here the effect of the quadratic and cubic in-plane displacements is small enough to be neglected. However, our 11DOF shell element provides a direct and natural way to include the effects of surface tractions when these are initially unknown (e.g. imposed in a metal forming problem), vary over the shell top and bottom surfaces and the shell thickness changes significantly. 


\section{Concluding remarks}

We presented in this paper a 4-node 3D-shell element that, in essence, represents a hierarchical improvement of the MITC4 shell element to include some important three-dimensional effects: the full 3D stress-strain law is used, the thickness change is included, shell tractions can be applied on the top and bottom surfaces of the shell, and the material can be incompressible. An important attribute is that the element does not result in undue ill-conditioning (as seen when shell surface top and bottom nodes are used in the kinematic description of 3D-shell elements) and does not result in instabilities (as seen with enhanced strain formulations).

There are various fields of applications for the 3D-shell element, including all those involving large strain effects and contact. But in particular, the given formulation provides the basic framework for effective large strain analyses using 3D orthotropic hyperelastic or elastic-plastic material descriptions [34-37] as encountered in bio-engineering and metal forming.

A quite outstanding research task is the mathematical analysis of the proposed 3D-shell element, as pursued in Refs. $[2,6,10,16,18]$ for other formulations.

\section{Appendix}

We consider the cantilever beam subjected to in-plane tractions described in Table 3, and use the Airy stress function method to solve for the stresses.

The stress equilibrium and strain compatibility conditions are satisfied by solving the equation:

$\frac{\partial^{4} \phi}{\partial x^{4}}+2 \frac{\partial^{4} \phi}{\partial x^{2} \partial z^{2}}+\frac{\partial^{4} \phi}{\partial z^{4}}=0$

where

$\tau_{x x}=\frac{\partial^{2} \phi}{\partial z^{2}}, \quad \tau_{z z}=\frac{\partial^{2} \phi}{\partial x^{2}}, \quad \tau_{x z}=-\frac{\partial^{2} \phi}{\partial x \partial z}$

with the following boundary conditions:

$\left.\tau_{x z}\right|_{z=\frac{t}{2}}=q_{t},\left.\quad \tau_{x z}\right|_{z=-\frac{t}{2}}=-q_{b}$

$\left.\tau_{z z}\right|_{z=\frac{t}{2}}=\left.\tau_{z z}\right|_{z=-\frac{t}{2}}=0$.

The trial stress function used is

$\phi=c_{0}\left(1+a_{1} x\right)\left(1+b_{1} z+b_{2} z^{2}+b_{3} z^{3}\right)$.

This function satisfies Eq. (A1).

Then the corresponding stresses are

$\tau_{x x}=\frac{\partial^{2} \phi}{\partial z^{2}}=c_{0}\left(1+a_{1} x\right)\left(2 b_{2}+6 b_{3} z\right)$,

$\tau_{z z}=\frac{\partial^{2} \phi}{\partial x^{2}}=0$,

$\tau_{x z}=-\frac{\partial^{2} \phi}{\partial x \partial z}=-c_{0} a_{1}\left(b_{1}+2 b_{2} z+3 b_{3} z^{2}\right)$.

From the force and moment equilibrium, the resultant shear force, axial force and bending moment at each section of the beam are

$V(x)=\int_{-t / 2}^{t / 2} \tau_{x z} \mathrm{~d} z=0$,

$N(x)=\int_{-t / 2}^{t / 2} \tau_{x x} \mathrm{~d} z=\left(q_{t}+q_{b}\right)(L-x)$

$M(x)=\int_{-t / 2}^{t / 2} \tau_{x x} z \mathrm{~d} z=\left(q_{t}-q_{b}\right)(L-x) \frac{t}{2}$.

Substituting Eq. (A5) into Eq. (A6) and applying the boundary conditions (A3), we obtain

$$
\begin{aligned}
& a_{1}=-\frac{1}{L}, \\
& c_{0} b_{1}=-\frac{\left(q_{t}-q_{b}\right) L}{4}, \\
& c_{0} b_{2}=\frac{\left(q_{t}+q_{b}\right) L}{2 t}, \\
& c_{0} b_{3}=\frac{\left(q_{t}-q_{b}\right) L}{t^{2}} .
\end{aligned}
$$

Therefore the solution is

$$
\begin{aligned}
\tau_{x x} & =(L-x)\left[\left(q_{t}+q_{b}\right) \frac{1}{t}+6\left(q_{t}-q_{b}\right) \frac{1}{t^{2}} z\right], \\
\tau_{z z} & =0, \\
\tau_{x z} & =\left[-\left(q_{t}-q_{b}\right) \frac{1}{4}+\left(q_{t}+q_{b}\right) \frac{1}{t} z+3\left(q_{t}-q_{b}\right) \frac{1}{t^{2}} z^{2}\right] .
\end{aligned}
$$

\section{References}

[1] Bathe KJ. Finite element procedures. New Jersey: Prentice Hall; 1996.

[2] Chapelle D, Bathe KJ. The finite element analysis of shells- fundamentals. Berlin: Springer; 2003.

[3] Dvorkin EN, Bathe KJ. A continuum mechanics based four-node shell element for general non-linear analysis. Eng Comput 1984;1:133-44.

[4] Bathe KJ, Dvorkin EN. A formulation of general shell elements - the use of mixed interpolation of tensorial components. Int J Numer Methods Eng 1986;22:697-722.

[5] Bathe KJ, Iosilevich A, Chapelle D. An evaluation of the MITC shell elements. Comput Struct 2000;75:1-30.

[6] Pitkaranta J. Mathematical and historical reflections on the lowest-order finite element models for thin structures. Comput Struct 2003;81:895-909.

[7] Bucalem ML, Bathe KJ. Higher-order MITC general shell elements. Int J Numer Methods Eng 1993;36:3729-54.

[8] Bathe KJ, Lee PS, Hiller JF. Towards improving the MITC9 shell element. Comput Struct 2003;81:477-89.

[9] Lee PS, Bathe KJ. Development of MITC isotropic triangular shell finite elements. Comput Struct 2004;82:945-62.

[10] Chapelle D, Bathe KJ. The mathematical shell model underlying general shell elements. Int J Numer Methods Eng 2000;48:289-313.

[11] Lee PS, Bathe KJ. Insight into finite element shell discretizations by use of the basic shell mathematical model. Comput Struct 2005;83:69-90.

[12] Bischoff M, Ramm E. Shear deformable shell elements for large strains and rotations. Int J Numer Methods Eng 1997;40:4427-49.

[13] Hauptmann R, Schweizerhof K. A systematic development of 'solid-shell' element formulations for linear and non-linear analyses employing only displacement degrees of freedom. Int J Numer Methods Eng 1998;42:49-69.

[14] El-Abbasi N, Meguid SA. A new shell element accounting for through-thickness deformation. Comput Methods Appl Mech Eng 2000;189:841-62.

[15] Klinkel S, Gruttmann F, Wagner W. A robust non-linear solid-shell element based on a mixed variational formulation. Comput Methods Appl Mech Eng 2006;195:179-201.

[16] Chapelle D, Ferent A, Bathe KJ. 3D-Shell elements and their underlying mathematical model. Math Models Methods Appl Sci 2004;14:105-42.

[17] Wall WA, Gee M, Ramm, E. The challenge of a three-dimensional shell formulation - the conditioning problem. Comput Methods Shell Spatial Struct, IASS-IACM; 2000.

[18] Chapelle D, Mardare C, Munch A. Asymptotic considerations shedding light on incompressible shell models. J Elast 2004;76:199-246.

[19] Fontes Valente RA, Alves de Sousa RJ, Natal Jorge RM. An enhanced strain 3D element for large deformation elastoplastic thin-shell applications. Comput Mech 2004;34:38-52.

[20] Vu-Quoc L, Tan XG. Optimal solid shells for non-linear analyses of multilaye composites - I. Stat Comput Methods Appl Mech Eng 2003;192:975-1016.

[21] Wriggers P, Reese S. A note on enhanced strain methods for large deformations. Comput Methods Appl Mech Eng 1996;135:201-9.

[22] Pantuso D, Bathe KJ. On the stability of mixed finite elements in large strain analysis of incompressible solids. Finite Elem Anal Des 1997;28: 83-104.

[23] Toscano RG, Dvorkin EN. A shell element for finite strain analyses: hyperelastic material models. Eng Comput 2007;24:514-35.

[24] Bathe KJ. ADINA System. Encyclopedia of Mathematics 1997;11:33-35 $<$ www.adina.com>.

[25] Betsch P, Stein E. An assumed strain approach avoiding artificial thickness straining for a non-linear 4-node shell element. Commun Numer Methods Eng 1995;11:899-909.

[26] Chapelle D, Ferent A, Tallec PL. The treatment of pinching locking in 3D-shell elements. Math Model Numer Anal 2003;37:143-58. 
[27] Sussman T, Bathe KJ. A finite element formulation for nonlinear incompressible elastic and inelastic analysis. Comput Struct 1987;26: 357-409.

[28] Bathe KJ. The inf-sup condition and its evaluation for mixed finite element methods. Comput Struct 2001;79:243-52.

[29] Chapelle D, Bathe KJ. Fundamental considerations for the finite element analysis of shell structures. Comput Struct 1998;66:19-36.

[30] Hiller JF, Bathe KJ. Measuring convergence of mixed finite element discretizations: an application to shell structures. Comput Struct 2003;81:639-54.

[31] Sussman T, Bathe KJ. Studies of finite element procedures - on mesh selection. Comput Struct 1985;21:257-64.

[32] Pantuso D, Bathe KJ. A four-node quadrilateral mixed-interpolated element for solids and fluids. Math Models Methods Appl Sci 1995;5:1113-28.
[33] Lovadina C. Analysis of strain-pressure finite element methods for the Stokes problem. Numer Methods Partial Differen Equat 1997;13:717-30.

[34] Montans FJ, Bathe KJ. Computational issues in large strain elasto-plasticity: an algorithm for mixed hardening and plastic spin. Int J Numer Methods Eng 2005;63:159-96.

[35] Montans FJ, Bathe KJ. Towards a model for large strain anisotropic elastoplasticity. In: Onate $\mathrm{E}$, Owen $\mathrm{R}$, editors. Chapter in computational plasticity. Springer-Verlag; 2007. p. 13-36.

[36] Bischoff JE, Arruda EM, Grosh K. A rheological network model for the continuum anisotropic and viscoelastic behavior of soft tissue. Biomech Modeling Mechanobiol 2004;3:56-65.

[37] Zhang Y, Dunn ML, Drexler ES, McCowan CN, Slifca AJ, Ivy DD, et al. A microstructural hyperelastic model of pulmonary arteries under normo- and hypertensive conditions. Ann Biomed Eng 2005;33:1042-52. 